\title{
A Valorized Scheme for Failure Prediction Using ANFIS: Application to Train Track Breaking System
}

\author{
Tse Sparthan1, Wolfgang Nzie1, Bertin Sohfotsing², Tibi Beda ${ }^{3}$, Olivier Garro \\ ${ }^{1}$ Department of Mechanical Engineering, National Advance School of Agro-Industrial Science, University of Ngaoundere, \\ Ngaoundere, Cameroon \\ ${ }^{2}$ Department of Mechanical Engineering, University Institute of Technology Fotso Victor, University of Dschang, Dschang, Cameroon \\ ${ }^{3}$ Department of Physics, Faculty of Science, University of Ngaoundere, Ngaoundere, Cameroon \\ Email: tseazoh@yahoo.com
}

How to cite this paper: Sparthan, T., Nzie, W., Sohfotsing, B., Beda, T. and Garro, O. (2020) A Valorized Scheme for Failure Prediction Using ANFIS: Application to Train Track Breaking System. Open Journal of Applied Sciences, 10, 732-757. https://doi.org/10.4236/ojapps.2020.1011052

Received: October 27, 2020

Accepted: November 24, 2020

Published: November 27, 2020

Copyright $\odot 2020$ by author(s) and Scientific Research Publishing Inc. This work is licensed under the Creative Commons Attribution International License (CC BY 4.0).

http://creativecommons.org/licenses/by/4.0/

\begin{abstract}
In the rolling stock sector, the ability to protect passengers, freight and services relies on heavy inborn maintenance. Initiating an accurate model suitable to foresee the change of attitude on components when operating rolling stock systems will assist in reducing lock down and favors heavy productivity. In that light, this paper showcases a suitable methodology to track degradation of components through the blinding of physic laws and artificial intelligent techniques. This model used to foresee failure deterioration rate and remaining useful life (RUL) speculation is case study to showcase its quality and perfection, within which behavioral data are obtained through simulated models initiated in Mathlab. For feature extraction and forecasting issues, different neuro-fuzzy inference systems are designed, learnt and authenticated with powerful outputs gained during this process.
\end{abstract}

\section{Keywords}

Failure Prediction (FP), Remaining Useful Life (RUL), Artificial Intelligence (AI), Traintrack System, ANFIS, Modeling

\section{Introduction}

The rolling stock industries are highly competitive, because the demand for railway transport is incomparable as it favors heavy means in transporting plenty of freights and passengers within long or short distances. Safety and security are great challenges that face when operating them. In the rolling stock sector, the ability to protect passengers, freight and services relies on heavy inborn 
maintenance strategy that focuses on the quality of the traintrack components. Since their design gives them alternative modes of working, it is difficult to follow them up using human knowledge. Initiating an accurate model suitable to foresee the change of attitude on traintrack components during field work will assist in dropping luck down and favor heavy productivity rate. Prognostic refers to an estimate of time to failure and risk, within which one or more existing and future failure modes on an asset are governed [1]. This makes it inevitable during preventive maintenance issues since it aims at eliminating dramatic luck down through RUL estimated and forecasting parameters. Prognostic is prominently split as model, data driven, experience oriented and hybrid (the combination of more than one distinct method) [2]. But due to congestion of components, inconsistency of field data, human comprehension extrapolated in practice, heavy attention on possible AI techniques to help prognostic issues are expected from technical and scientific viewpoints.

The expected activity here is to establish a failure forecaster and an RUL estimator that is consistent with issues of heavy field data, low cost, phase change of components and thus capable of directing prognostic activities on new and existing systems with human comprehension. These are negative motivations of the data and experience oriented methods especially when focused on new process line, as it grants an extra appreciation to model oriented methods as it focuses on fixed threshold data and physics of the system.

During design, each train-track component is associated with one or more physics comprehension which favors the stability of forecasting the growing deterioration rate using model oriented methods even though in most cases majority of component attitudes are nonlinear. To counter the nonlinearity issues, many authors have valoralised the use of AI ideas center on neural network, fuzzy logic, Markov Models, particle filtering and other genetic algorithms [3] [4]. Excessive usage of time during execution and as such lack of human comprehension due to their hidden attitude are their negative motivation when relying on them. And thus the actual status of the system mostly relies on the past. Meaning a hybrid prognostic system that has the ability to deal with the past, present and future status of the system needs to be initiated. The hybrid neuro-fuzzy inference system showcases a safe usage because of its accuracy and less time consumption even though it requires a heavy architecture. As a soft computing tool, ANFIS showcases high standard in modeling input/output nonlinear issues [5]. Moreover, none of the above authors has insisted on the use of ANFIS in estimating and to foresee RUL issues on train track components in short or long terms.

A hybrid fault discovery and segregated unit that blind model oriented for proactive issues with ANFIS and data oriented for active validation was introduced in [6]. The hybrid diagnoser not only showcases the robustness and ability to discover and extract multitudes of unpleasant scenarios but thus showcases a high selectivity and sensitivity attitude since it can answer rapidly when there is complex modification of the supposed system. But the view point of this paper is 
centered on initiating a hybrid prognostic unit that will automatically deduce the RUL of traintrack or other process lines accurately and faster. The remains of this work entail: the philosophy of ANFIS to fault prognostic issues in section two, the proposed methodology established on failure degradation rate and RUL forecasting in section three, application of this new model in four, finally conclusion and future contributions in five.

\section{ANFIS for Fault Prognostics}

Neural networks favor precise timing and speed recognition, and are distinguished from static to dynamic when applied to nonlinear problems. Feed-Forward, Multi-Layer Perceptron, Radial Basis Function, General Regression Neural, Recurrent and Time Delay Neural Networks are identified. They face transparency issues as declaration of their results concern human comprehension because of their hidden attitude. The elimination of black box issues favors the use of fuzzy logic that uses excess timing and low quality output. To stabilize these two orderly learning systems, both of them are blind to form a neuro-fuzzy inference system (ANFIS) which discards their wrong issues and favors the good one. ANFIS used in RUL forecasting and future usage parameters of any industrial devices are distinguished from Takagi Sugeno Kang (TSK) and Mamdani system. Figure 1 examines the attitude of a Sugeno system whose output is either a linear or constant quantity. Made of five layers, the TSK model constitutes an antecedent unit which performs fuzzification and If then rules combinations through the optimization of its membership function using gradent decent methods in a backword manner. To round up, layer three serves as the unit for membership normalization, layer four for if then statement and lastly layer five for output computational aggregation. The learning in these layers is updated using least square methods in a forward manner [5].

\section{Methodology}

\subsection{The Proposed Hybrid System}

In reality, the attitude of every engineering system differs even if they are governed with identical or different physics laws. Maintenance strategies centered on lock down before reinstatement to normal status are no longer favoured because they don't have the capacity to track the phase change of individual component as time thus modifies [7]. To foresee the evolution of their various attitudes during usage is aspiration of attentiveness for company owners, engineers and scientists. Developing a novel prognostic methodology which combines proactive and active status of traintrack systems is inevitable, because understanding the status of individual components during usage is cost effective as far as maintenance issues are concerned. Meaning, their sudden lock down can only be followed up directly through the knowledge of degradation centered on the attitude of the components, its purpose and external environmental factors which art as barriers for smooth operation. 


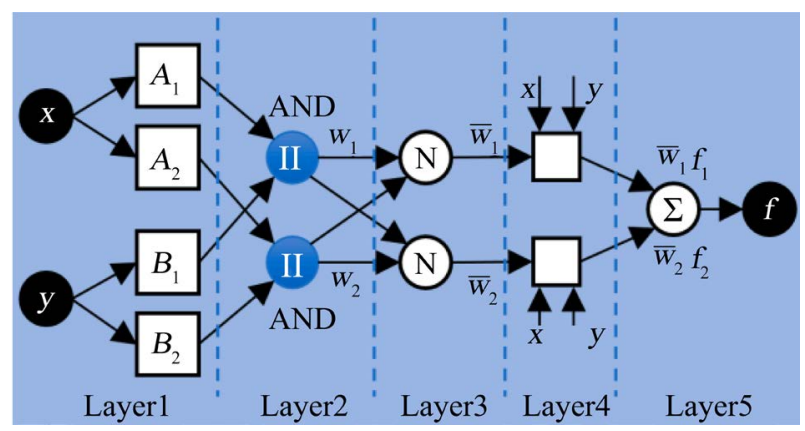

Figure 1. Architecture of adopted ANFIS for forecasting [5].

This calls for an intelligent base maintenance tool that blind analytical models define by physics laws and long term history of the system demanding. However, when the knowledge of system components is known physical, prognostic issues are easily solved due to its flexibility of adapting on phase change situations and thus RUL component calculation and forecasting. Figure 2 is the segregated procedure that blind physics laws and AI techniques (ANFIS) to outperform failure estimation and RUL forecasting issues on complex, linear and nonlinear system. It is centered on quick failure discovery and extraction quantities favorable to track efficiently the rate of deterioration. Follow up and modeling the rate of deterioration strictly relies on quick discovery and analysis as they play good contributions in quantification and safe life forecasting. Even though many have focused on statistics and after failure data sets gained from field work, the presence of uncertainties in most cases weakens the quality of the data collected and as such pushes them to use physics laws. If during initiating a new train track system, we define the healthy state using physics Laws of each component, and secondly have the ability to store its historical information qualitatively during the usage phase of the said system, then a favorable time to failure tool is required to fight against sudden break down.

Figure 2 segregates the automatic prognostic procedure in six unidentical modules for remaining safe life future recognition for engineering systems.

Process recognition module: This brings forward a legit model of the said system under study considering the attitude of individual components, their missions and lastly external environmental parameters that affect the smooth running of the entire system. The module is segregated in three aspects within which a graphical model demonstrating how the entire attitude of system components are inter related, the construction of a physics model either through mathematical or using soft computing tools designed, for real time analysis. Next is to extract detail the running data's that characterizes the fair and unfair mission of the system through Component Individual Parameters Identification Strategy (system design hand book). Finally, identify and initiate within the system model the degradation parameter influenced either by external environmental issues or phase changes of the system components with respect to time evolution rate. 


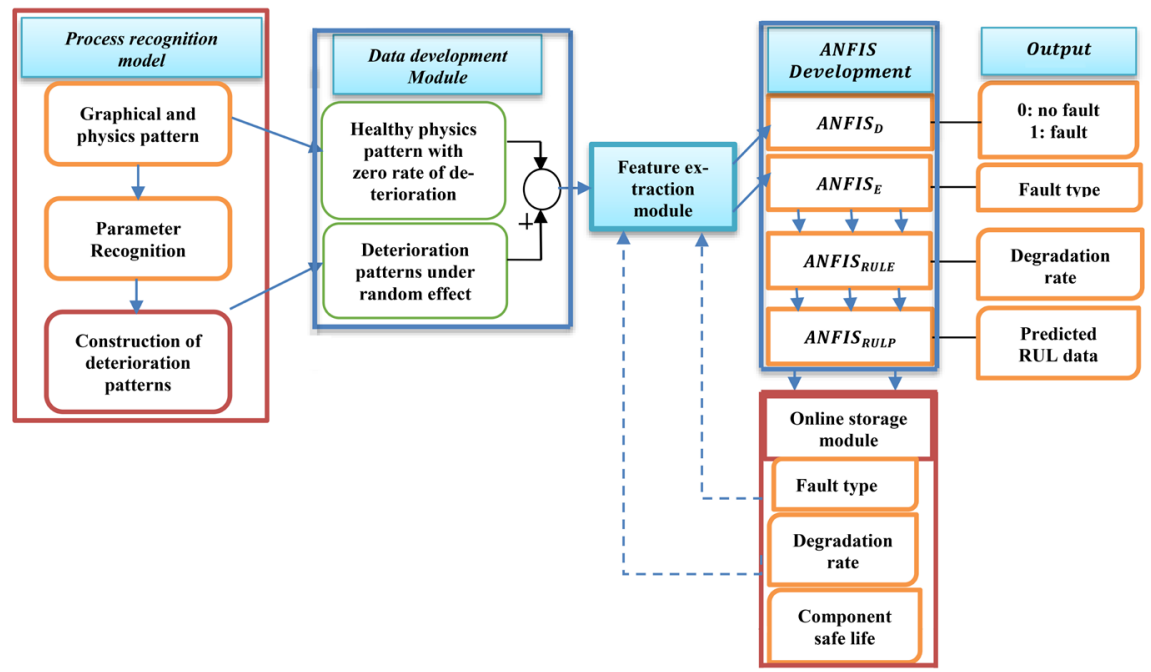

Figure 2. An automatic hybrid model for RUL prediction.

Data development module: Since in recent cases, most systems are nonlinear and have a stochastic attitude during their usage phase, this module is segregated for active and proactive activities. For systems already in use with no information provided by manufacturers, the system model is simulated in the absence of deterioration in order to quantify a threshold data. For new ones, the manufacturers must provide an input threshold data which defines the maximum and minimum rate of degradation. These threshold signals which define the quality of the system during operations is then used by the initiated predictor to derivate at every given instant the deterioration rate obtained under unstable conditions. The change obtained when the system is deterioration free and when it starts to deteriorate is considered as residual data within which they are further consumed by the feature extraction module to develop residual estimated signals.

Feature Extraction Module: This module is made of two post processing sections, which make it differ from literature. In the first case, feature extraction activities are centered on the unlike measured variables and their approximate called residual signal which carries reliable information necessary to estimate the rate of deterioration based on the input/output model of the system. The second case relies on signals obtain after a given period of operating the said system. The operational historical information acquired within a certain period store in the online storage module is sent back to the feature extraction module. Before then, all unpleasant data's must be distinguished from fairly ones. New residual signals are obtained based on the comparison between the non-deteriorating and the deteriorating signals (Figure 3). The manufacturing of such module permit the elimination of data shortages or loss as forecasting is concerned during intelligent maintenance practices on train track units. As an auto verification tool, the output of this module is linked to the ANFIS's modeling units and the display module for model validation if necessary. Note that the quality of the residual signal in both situations relies on linear and nonlinear least squares algorithms as threshold data are easily obtained from physics laws. 


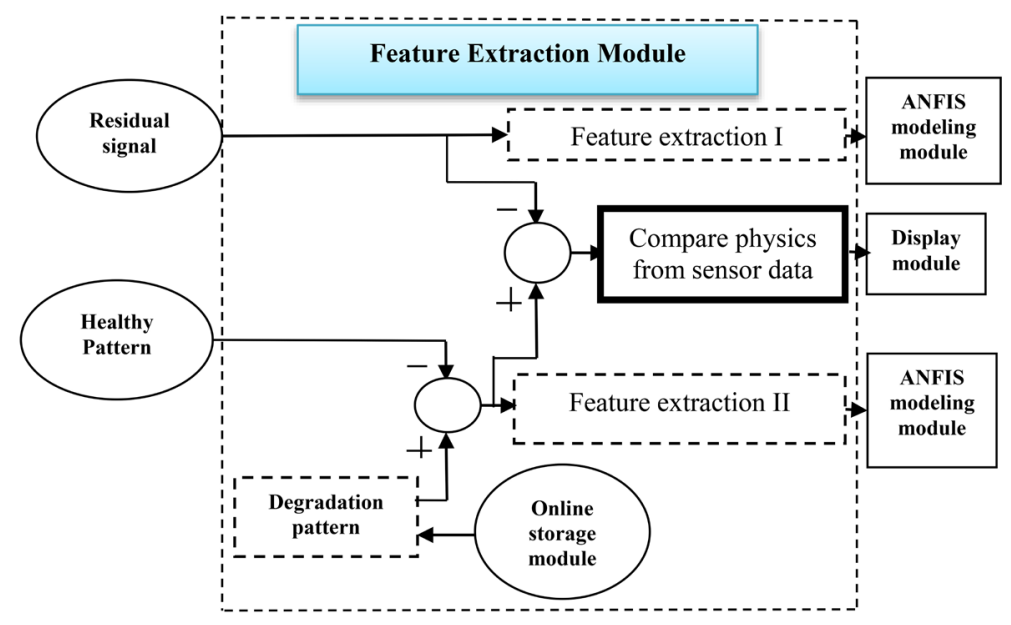

Figure 3. Internal view of feature extraction module.

ANFIS Modeling Module: The ability of this module is strongly centered on the quality of the recommended signals obtained from the feature estimation module. Each ANFIS system Operates both in series and parallel (Figure 2): ANFIS $_{\text {D\&E }}$ identifies the type of degradation rate as they are designed to discover and extract faults irrespective of their mode of occurrences using an online damage estimator. Due to nonlinearity attitude, each component or subsystems will have its own damage signature which must be segregated at the early stage. ANFIS $_{\text {RULE }}$ tracks and quantifies the rate of damage for each components or subsystems within a short interval directly. The damage value obtained at this stage corresponds to the amount of deterioration for the specific component. To foresee the remaining safe life of the said component or system, a long term forecaster $\left(\mathrm{ANFIS}_{\text {RULP }}\right.$ ) which has the ability to predict until a predetermined value that corresponds to the threshold value defined in process recognition module is gained. ANFIS $S_{\text {RULP }}$ is recommended because it is difficult to have a genuine knowledge about the phase change of the system and thus, if their safe life values may meet their failure indicator index (Condition monitoring index) when needed. An advantage of segregating this module permits an accurate step by step monitoring of the traintrack system as each activity can be visualized by the operator.

Display Module: The status of the train track system is outputed by this module. The output visualizes the good or break down scenarios. Each ANFI$\mathrm{S}_{\text {output }}$ is connected to this module to interpret each condition. For damage to be localised, output shall indicate zero conditions for no degradation, one for possible detected degradation and finally the given component and its mode of degradation (value). The damage phase change is thus estimated and visualized in this module using numerical values and linguistic rules for easy comprehension and understanding (good, fair and close to failure, complete damage) to define the mode at which the components are for quick maintenance interventions. The conditions of the system should be defined by the operator. 
Online Storage Module: The output information of the systems attitude are collected on regular bases and stored online in the form of data's. These real time signals that interpret and mitigate the status of the system are directly used as input signals in the feature extraction module after the system must have worked for a given period. The aim here is to verify the quality of the prognostic tool through comparism of the model oriented and the historical signal methods because the safe life parameter drops continuously as the rate of damage improve. Thus the attitude of each component portray its own style of damage and requires a certain pattern to track its degradation rate.

\subsection{Manifestation of the Prognostic Procedure}

The quality and accuracy of damage management in rolling stock components rely on the manner of which their degradation rates are being monitored. Early failure discovery should occur from the first instance of degradation as they can be used in tracking and forecasting safe life of the system. However, the attitude of damage in train-track components differs and as such requires its own degradation pattern. The proposed segregated prognostic approach is flexible as it focused on the given system and it damage parameter at a time. The mechanical failures in the rolling stock systems associate $44.7 \%$ to wheelset, $36.7 \%$ to braking and $18.6 \%$ to the chassis. Brake deterioration indirectly account for $75 \%$ of above $100 \%$ failures [8]. The braking operation is critical and must be monitored on countless bases. Estimating and foreseeing the safe life of its components during usage will reduce sudden failure and as such improve the safety of freights and passengers. Pressure drops within pipelines due to leaks and Loss of contact between the lining/disc under the effect of wear are the two mechanical failures encountered during braking. The next portion of this document showcases how the second failure mode uses wear as degradation parameter to track and monitor the safe life of the train braking operation affected by external impurities in the environment. Figure 4 is the full suggested model that uses material removal as real time indicator to track damage of the rail vehicle lining/disc system. Feature extraction signals are segregated from model $\left(\% \tau_{Z U}\right)_{1}$ to data $\left(\% \tau_{Z U}\right)_{2}$ and physics/sensor $\left(\% \tau_{Z U}\right)_{3}$ oriented for ANFIS model validation and residual signals quality control.

\subsubsection{Process Recognition Module}

Breaking actions are inevitable since they are used for speed reduction or complete stoppage of the train. Their safe operation and acoustic emission are centered on the contact condition within the disc and lining. Efficient braking is registered under dry contacts scenarios within which kinetic energy is transformed to thermal energy. These phase changes lead to vibration, fatigue and thus removal of material due to thermal or mechanical stresses, unstable contact pressure/region, and temperature growth [9] [10] [11] [12]. Material removal increases the penetrating distance and as such influences the contact condition of the lining/disc. Figure 5, Figure 6 is an eyeshot and the suggested model of a 


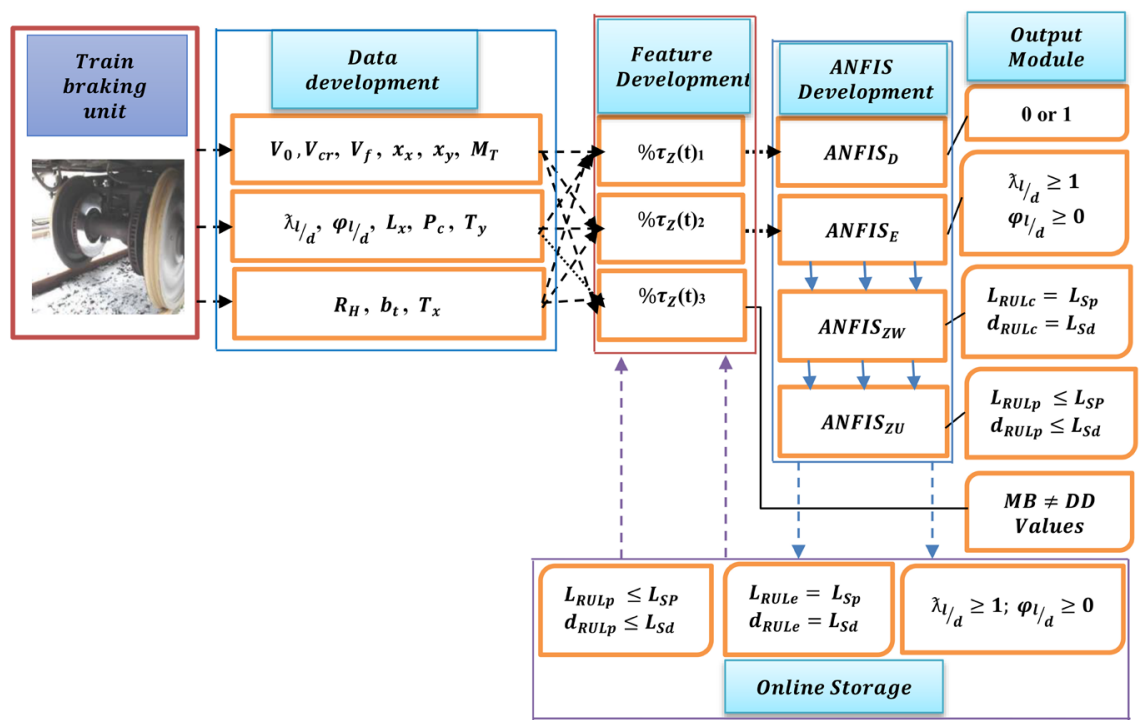

Figure 4. Full suggested ANFIS model for damage tracking of lining/disc system.

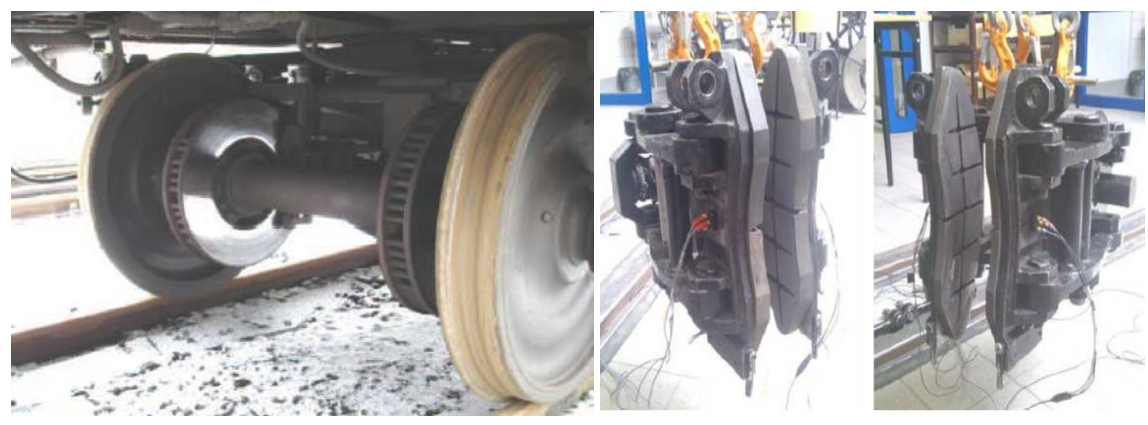

Figure 5. Eyeshots of the high speed axel mounted lining/disc brake unit.

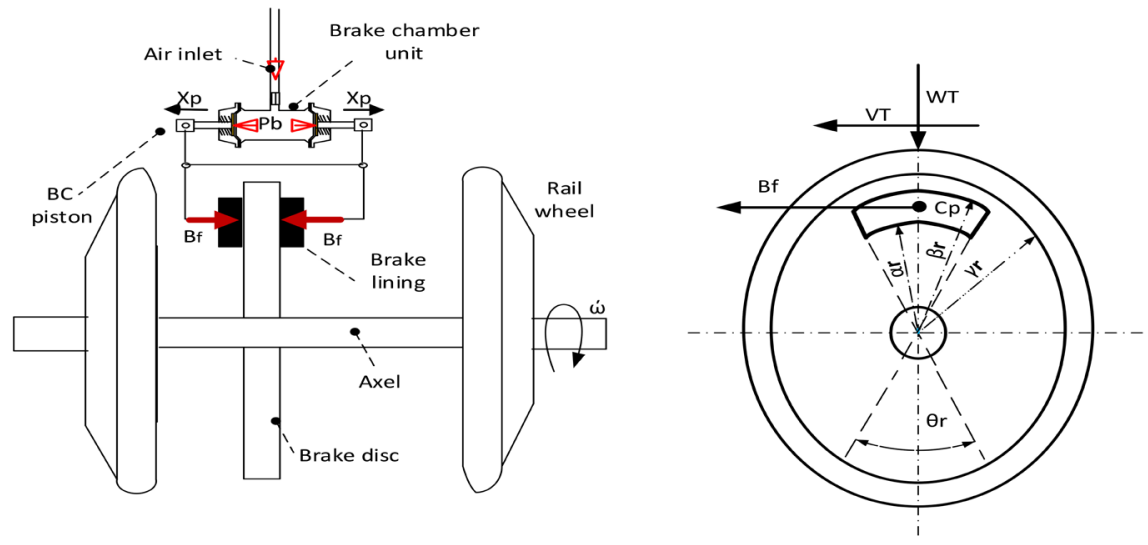

Figure 6. Model of the high speed axel mounted disc brake.

high speed axel mounted disc braking unit commonly used in the Cameron railway industry (CAMRAIL). They are made of a frictional ring and interconected hubs. As compressed air arrives the brake chamber, the outword movement of the piston closes the brake caliper and thus iterates the braking effect through the lining/disc contact. The axel receives and transfers consistently the 
brake effect from the disc to its respective wheels in order to slow or stop the train. Many authors have invested their time both in theory and field evaluation of wear attitudes and their effect on rail, automobile and manufacturing industries. Issues on vibration, speed, cracks, brake forces, contact pressure, temperature, frictional coefficient related to material removal has been investigated under dry and wet environment [13] [14] [15] [16] [17]. Their analysis segregates the brake lining/disc material removal during field operation in two categories

Breaking actions are inevitable since they are used for speed reduction or complete stoppage of the train. Their safe operation and acoustic emission are centered on the contact condition within the disc and lining. Efficient braking is registered under dry contacts scenarios within which kinetic energy is transformed to thermal energy, a model that uses energy perspectives to showcase the behavior and working mode of the braking lining/disc without the reality of worn. Secondly, models that bring together the rate of material removal, working parameters and energy generated during braking mode of the train without safe life tracking under different wear conditions. Even though these approaches provide high esteem knowledge about the attitude during field mode of the lining/disc, it is important to bring forth a model that can be used to investigate the rate of material removal and evaluate the safe life of engineering systems (lining/disc under different wear conditions: adhesive, abrasive and fatigue) and thus motion and environmental factors. Due to the continuous usage of the train, braking is performed both in the dry and wet scenarios. The wet condition influences it quality as the frictional coefficient reduces. The physics attitude that iterates the lining/disc dynamic contact scenario with time dependence under both conditions where; $l / d$ lining/disc, $\lambda_{l / d}$ clearance between the lining and the disc before braking, $\Delta(t)$ penetrating distance during applied and hold phase of the brakes, $X_{l / d}$ deflection due to viscoselastic nature of their material of construction (Equation (2)), $\varphi_{l / d}$ is material removed during the apply and hold phase of the brakes (Equation (3)). The model equation thus integrate external factors (motion factor $\rho_{m}$ and environmental factor $\rho_{E}$ ).

$$
\begin{array}{r}
\lambda_{l / d}(t)+X_{l / d}(t)+\varphi_{l / d}(t)=\Delta(t) \\
X_{l / d}(t)=\phi_{r} \delta_{l / d} w_{l / d}^{\prime} P_{c}(t) ;\left\{\begin{array}{l}
\delta_{l / d}=\frac{H_{l / d}\left(1-V_{T}^{2}\right)}{\pi E_{l / d}} \\
w_{l / d}^{\prime}=\frac{\theta_{r}}{4}\left(1-\frac{\alpha_{r}}{\beta_{r}}\right)
\end{array}\right. \\
\varphi_{l / d}(t)=\rho_{E} \rho_{m}\left(K_{l / d}\right)_{m} \int_{0}^{T} \in_{l / d}(t) d(t) \\
\in_{l / d}(t)=\left(P_{c}^{\alpha}(t) \cdot V_{c}^{\beta}(t)\right)_{l}+\left(P_{c}^{\alpha}(t) \cdot V_{c}^{\beta}(t)\right)_{d} \\
P_{c}=\left(\frac{C_{p}}{H}\right)_{l / d} ; V_{c}=\left(\frac{V_{T}}{V_{c r}}\right)_{l / d} ; V_{c}=\frac{\left(V_{0}+V_{f}\right) \beta_{r} \theta_{r}\left(1-\frac{\alpha_{r}}{\beta_{r}}\right) \sqrt{r_{w}}}{14.4 \alpha_{w} V_{c r}}
\end{array}
$$




$$
\rho_{m}=\frac{K_{0} x_{x} x_{y}}{M_{r} x_{x} x_{y}+M_{T}} \text { and } \rho_{E}=R_{H} C^{\prime}+m_{d}
$$

$C_{p}$ contact pressure, $\phi_{r}$ Love displacement of the lining/disc [15], $E_{l / d}$ elastic modules, $H_{l / d}$ material hardness, contact angle, $V_{T}$ and $V_{c r}$ train initial sliding and critical braking velocities (highest damping effect of the lining), $\alpha_{w}$ wheel radius, $\alpha_{r}$ and $\beta_{r}$ are the respective internal and external radius, $V_{0}$ train initial sliding speed before brake is applied, $V_{f}$ sliding speed after braking, $x_{x}$ length of the rail road, $x_{y}$ width of the rail road, $M_{T}$ train mass, $M_{r}$ mass of the rail, $K_{0}$ stiffness of the rail road, $R_{H}$ relative humidity, $C^{\prime}$ lining factor and $m_{d}$ dust particle, $\rho_{l}$ density. $K_{l / d}$ the wear coefficient of the lining/disc and thus used to segregate from abrasive (ab), adhesive (ad) and fatigue (f) wear types (Equation (5)) [17].

$$
\begin{gathered}
\left(K_{l / d}\right)_{m}=\left(K_{l}+K_{d}\right)_{m} \\
\left(K_{l / d}\right)_{a d}=\left(\partial \frac{\Delta \vartheta_{l / d} \cdot H_{l / d}}{W_{T}}\right)_{a d} ; \\
\left(K_{l / d}\right)_{a b}=\left(\frac{\Delta \vartheta_{l / d} \cdot H_{l / d}}{W_{T} \cdot L_{x}}\right)_{a b} ; \\
\left(K_{l / d}\right)_{f}=\left(\frac{3 \sqrt{3} \cdot r_{p} \cdot \mu_{l / d}}{C^{D} \cdot \Delta \gamma_{s}^{1-D}}\right)_{f} \\
\mu_{l / d}=\frac{I_{r} \cdot \omega_{r}-B_{m} \cdot b_{t}}{2 P_{f} \cdot b_{t} \cdot \alpha_{r e f}}
\end{gathered}
$$

With $I_{r}$ wheel moment of inertia, $\Delta \vartheta_{l / d}$ volume of removed material for the lining or disc, $\partial$ degree of wear (0: no wear \& 1: over wear), $W_{T}$ the load, $L_{x}$ train sliding distance, $r_{p}$ plastic/sliding working ratio, $\mu_{l / d}$ frictional coefficient, $C$ and $\Delta \gamma_{s}$ monotonic and shear strains, $D$ is the Mansor constant, $\omega_{r}$ train angula velocity, $b_{t}$ braking time, $B_{m}$ wheel frictional moment, $P_{f}$ piston force, $\alpha_{\text {ref }}$ lining/disc contact radius. Practically, the lining/disc wear is stochastic and can be segregated in three scenarios: new, usage and failure. For new scenario (zero wear), Equation (1) iterates:

$$
\lambda_{l / d}(t)+\frac{\phi_{r} \cdot H_{l / d} \cdot \theta_{r} \cdot\left(1-\frac{\alpha_{r}}{\beta_{r}}\right) \cdot\left(1-V_{l / d}^{2}\right)}{4 \cdot \pi \cdot E_{l / d}} \cdot P_{c}(t)=\Delta(t)
$$

For usage and failure scenarios (material removal), Equation (1) iterates

$$
\begin{aligned}
& \lambda_{l / d}(t)+\frac{\phi_{r} \cdot H_{l / d} \cdot \theta_{r} \cdot\left(1-\frac{\alpha_{r}}{\beta_{r}}\right) \cdot\left(1-V_{l / d}^{2}\right)}{4 \cdot \pi \cdot E_{l / d}} \cdot P_{c}(t) \\
& +\left(K_{l}+K_{d}\right)_{m} \cdot \frac{K_{0} \cdot x_{x} \cdot x_{y}\left(R_{H} C+m_{d}\right)}{M_{r} x_{x} x_{y}+M_{T}} \int_{0}^{T}\left(P_{c}^{\alpha}(t) \cdot V^{\beta}((t) d(t))_{l / d}=\Delta(t)\right.
\end{aligned}
$$

In reality, Equation (4) iterates that there exist three kinds of material removal 
attitude during usage phase. These attitudes can be blind to transform Equation (1) to a universal matrix model as;

$$
\begin{aligned}
& {[A]-[B][P]=E U} \\
& A=\left[\begin{array}{ccc}
\Delta-\left(\lambda_{l}+\lambda_{d}\right) & 0 & 0 \\
0 & \Delta-\left(\lambda_{l}+\lambda_{d}\right) & 0 \\
0 & 0 & \Delta-\left(\lambda_{l}+\lambda_{d}\right)
\end{array}\right] \text {; } \\
& B=\left[\begin{array}{ccc}
\phi_{r} \delta_{l / d} w_{l / d}^{\prime} & 0 & 0 \\
0 & \phi_{r} \delta_{l / d} w_{l / d}^{\prime} & 0 \\
0 & 0 & \phi_{r} \delta_{l / d} w_{l / d}^{\prime}
\end{array}\right] \\
& U=\left[\begin{array}{ccc}
\left(\varphi_{l}+\varphi_{d}\right)_{a d} & 0 & 0 \\
0 & \left(\varphi_{l}+\varphi_{d}\right)_{a b} & 0 \\
0 & 0 & \left(\varphi_{l}+\varphi_{d}\right)_{f}
\end{array}\right] \\
& P=\left[\begin{array}{lll}
P_{c 1} & P_{c 2} & P_{c 3}
\end{array}\right]^{\mathrm{T}} \text { and } x=\left[\begin{array}{ll}
P_{c}(t) & \dot{P}_{c}(t)
\end{array}\right]^{\mathrm{T}}
\end{aligned}
$$

where $E$ is an identical 3 by 3 matrix, $U$ the degradation matrix, $P$ the output matrix and $x$ the system state quantity gains after a time step differentiation of Equation (8) in first order (Equation (9)).

$$
\begin{gathered}
H \dot{P}_{c}(t)+K P_{c}(t)=\dot{\Delta}^{\prime}(t) \\
H=\phi_{r} \delta_{l / d} w_{l / d}^{\prime} ; \alpha=\beta=1 \\
K=\left(K_{l / d}\right)_{m} \rho_{E} \rho_{m} V_{c} ; \dot{\Delta}^{\prime}(t)=\dot{\Delta}(t)-\dot{\lambda}_{l / d}(t)
\end{gathered}
$$

The actual attitude of the train lining/disc relies on the characteristic information showcase in table one below. The lining constitutes Becorit B36 material and the disc of gray cast iron.

\subsubsection{Data Development Module}

The chiping of material affects the contact pressure and thus acts as a disturbance for uniform pressure build up between the lining/disc. During field operations, it is very difficult to deduce the contact pressure per contact point as the braking operation is very fast [15] The contact pressure is assumed to be uniform at every point along the lining/disc and as such influence the contact regime to be in a non-variable situation (Table 1).

Equation (9) can be resolved using ODE45 in Mathlab to gain the contact pressure evolution at every given velocity and time steps $\left(P_{c}(t)\right.$ and $\left.P_{c}(V)\right)$ Figure 7, where $t$ is considered as the braking time of the train $\left(t=b_{t}\right)$. From Figure 6 , an increment in train speed directly affects the duration of slow down or complete stoppage. Though, once the contact pressure is calculated, the wear rate can be tracked under different wear scenarios using equation (1\&4) as showcased in Figures 8-10.

The stability and increment of the contact pressure differs under dry and wet braking and as such has a very high impact in evaluating the lining/disc wear 
scenarios during field operations. When the lining/disc contact is dry, the contact pressure required to slow/stop the train is small because the friction is high and almost constant (Figure 6), thus the rate of material removal from the lining/disc is drastically low and uniform. Under wet conditions, the frictional coefficient is low and unstable. Material removal in this case is high as the contact pressure and distance required to stop/slow the rail vehicle is drastically increased (Figures 8-10).

Table 1. Actual system operational parameters [18].

\begin{tabular}{cccccc}
\hline Ref & Designation & Ref & Designation & Ref & Designation \\
\hline$\lambda_{l / d}$ & $(1 \times 2) \mathrm{mm}$ & $\alpha_{r}$ & $207 \mathrm{~mm}$ & $V_{T}$ & $340 \mathrm{~mm}$ \\
$\beta_{r}$ & $297 \mathrm{~mm}$ & $\alpha_{r e f}$ & $207 \mathrm{~mm}$ & $H_{d}$ & $195^{ \pm 5} \mathrm{HB}$ \\
$K_{0}$ & $150.88 \times 10^{7} \mathrm{Nm}$ & $x_{x}$ & $247 \mathrm{~mm}$ & $E_{d}$ & $103 \mathrm{GPa}$ \\
$M_{r}$ & $56 \mathrm{~kg} / \mathrm{m}$ & $M_{T}$ & {$[600$ to 1000$] \mathrm{mm}$} & $\rho_{d}$ & $7155 \mathrm{~kg} / \mathrm{m}^{3}$ \\
$m_{d}$ & $20 \mu \mathrm{m}$ & $C_{1}$ & $1.705 \times 10^{4} \mathrm{~kg}$ & $e_{d}$ & $110 \mathrm{~mm}$ \\
$R_{H}$ & {$[20 \mathrm{to} 90] \%$} & $\alpha_{w}$ & 2 & $M_{d}$ & $120 \mathrm{~kg}$ \\
$C^{\prime}$ & 1 & $V_{T}$ & $340 \mathrm{~mm}$ & $D$ & 2 \\
$H_{l}$ & $111^{ \pm 0} \mathrm{HB}$ & $e_{l}$ & $35 \mathrm{~mm}$ & $I_{r}$ & $14 \mathrm{~kg} / \mathrm{m}^{2}$ \\
$E_{l}$ & $2.6 \mathrm{GPa}$ & $M_{l}$ & $30 \mathrm{~kg}$ & $B_{m}$ & $4.02 \mathrm{~N} / \mathrm{m}$ \\
$\rho_{l}$ & $2045 \mathrm{~kg} / \mathrm{m}^{3}$ & & & & \\
\hline
\end{tabular}
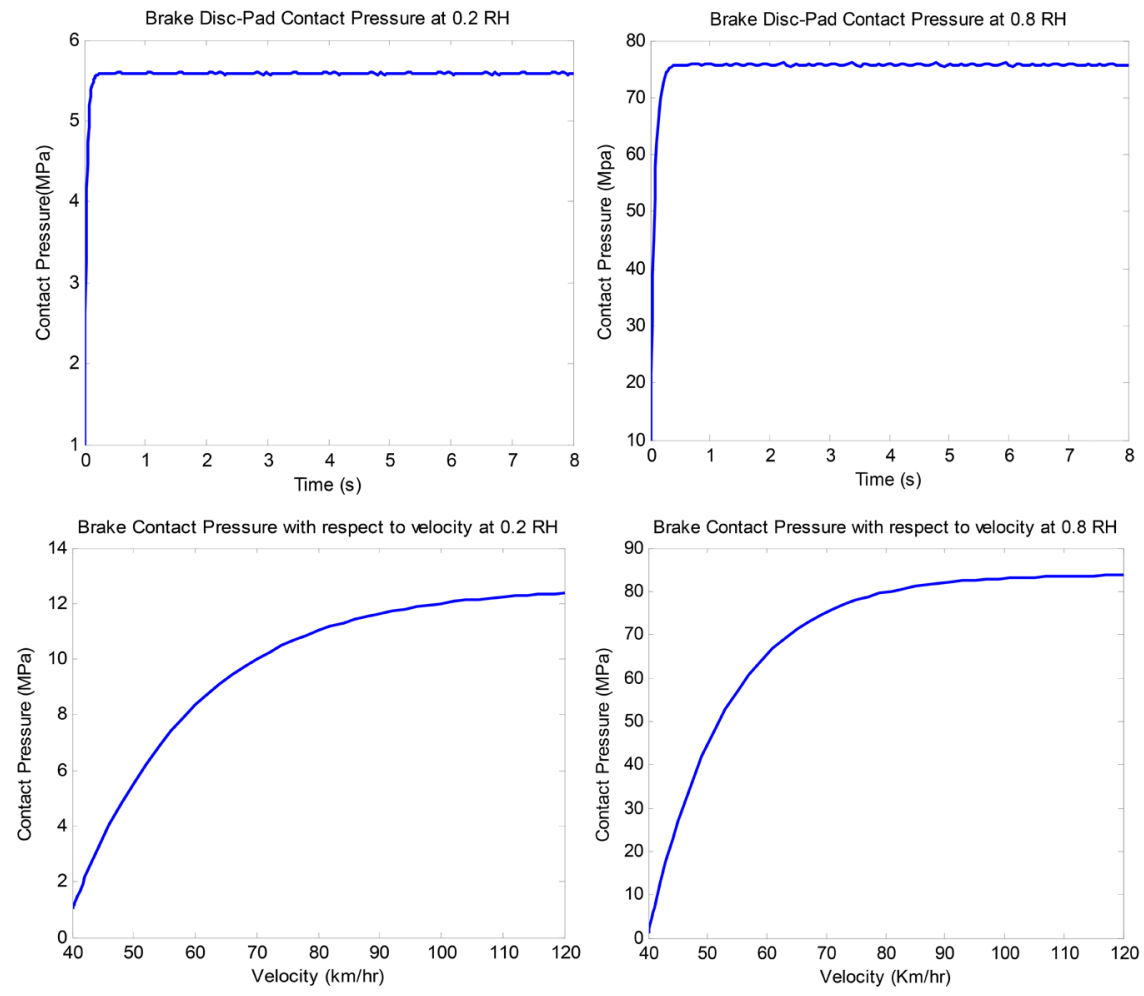

Figure 7. Evolution of $P_{c}$ (time, velocity) under dry and humid braking scenarios. 
The average frictional coefficient thus relies on the status of the lining (mode of material removal). An increment in material removal of the lining reduces the average characteristics of the frictional coefficient even though the braking speed at the start is greatly influential as compare to the contact pressure. The presence of oxygen and moisture obtained from the environment at the start of braking act as a lubricant between the lining/disc dry contact, their rate of material removal is very slow as the films created due to friction are generated to gain stability.

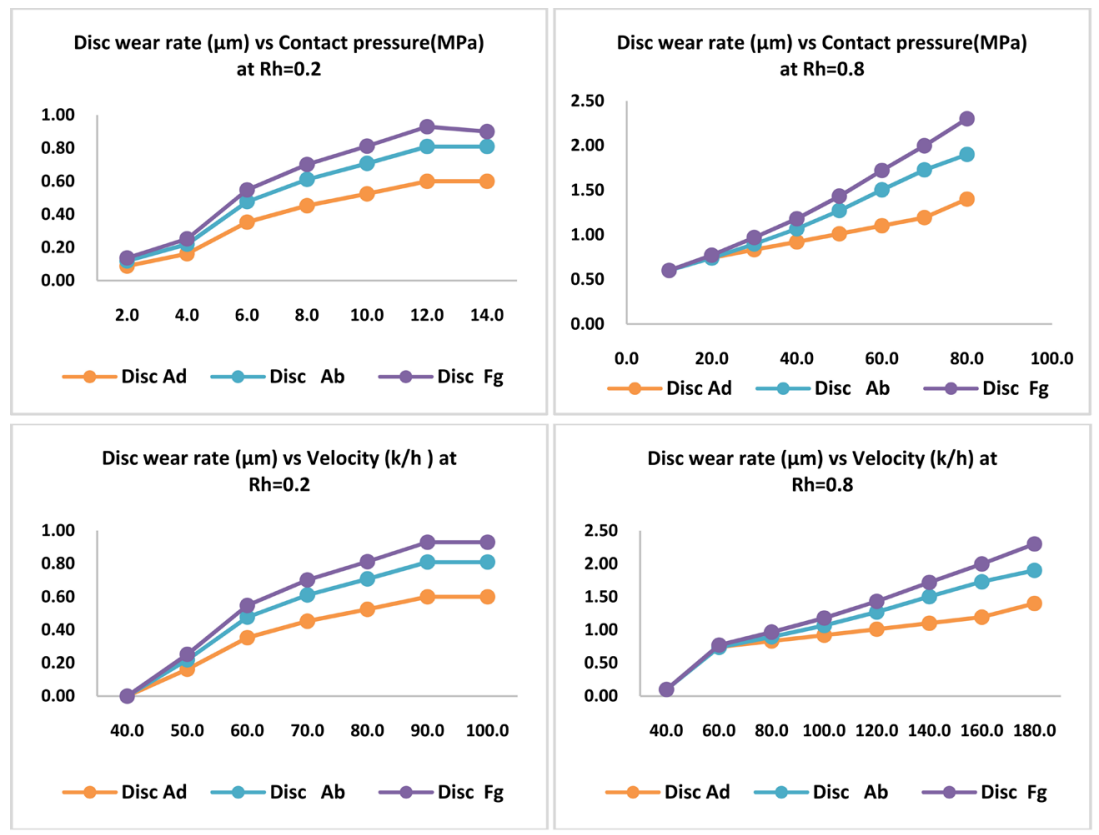

Figure 8. Disc rate of material removal during dry and wet braking scenarios.

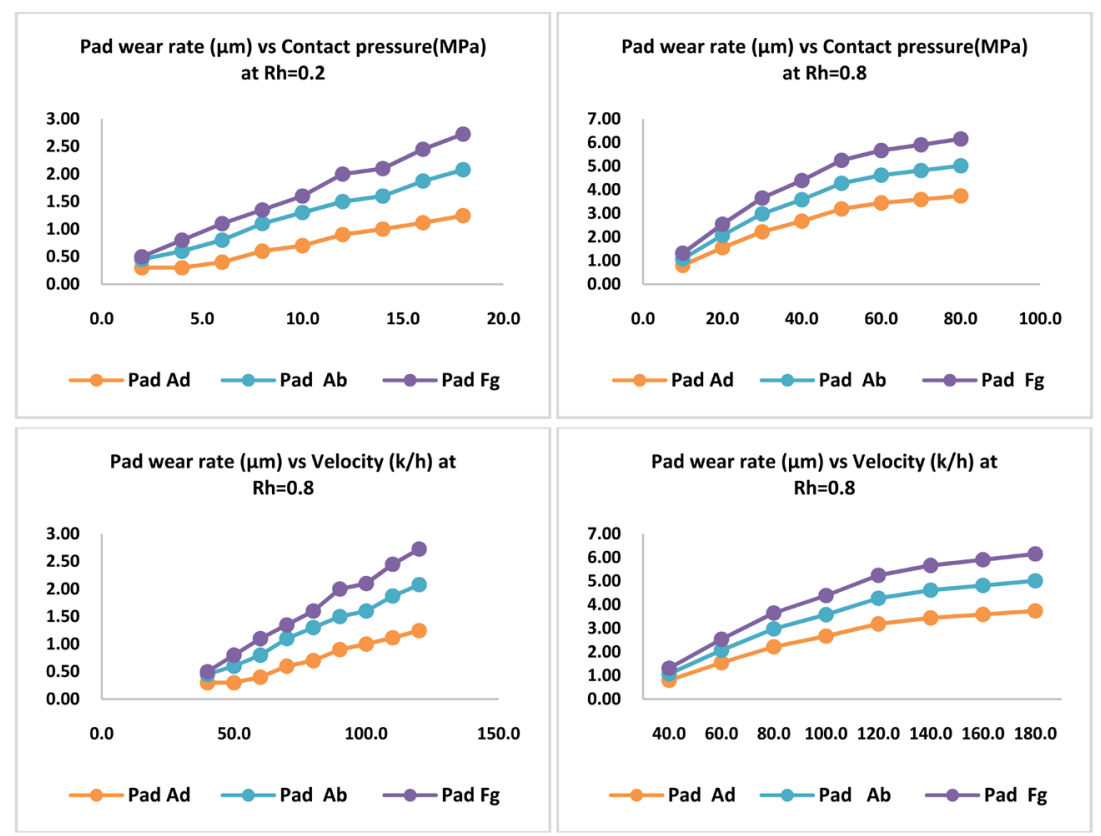

Figure 9. Lining rate of material removal during dry and wet braking scenarios. 


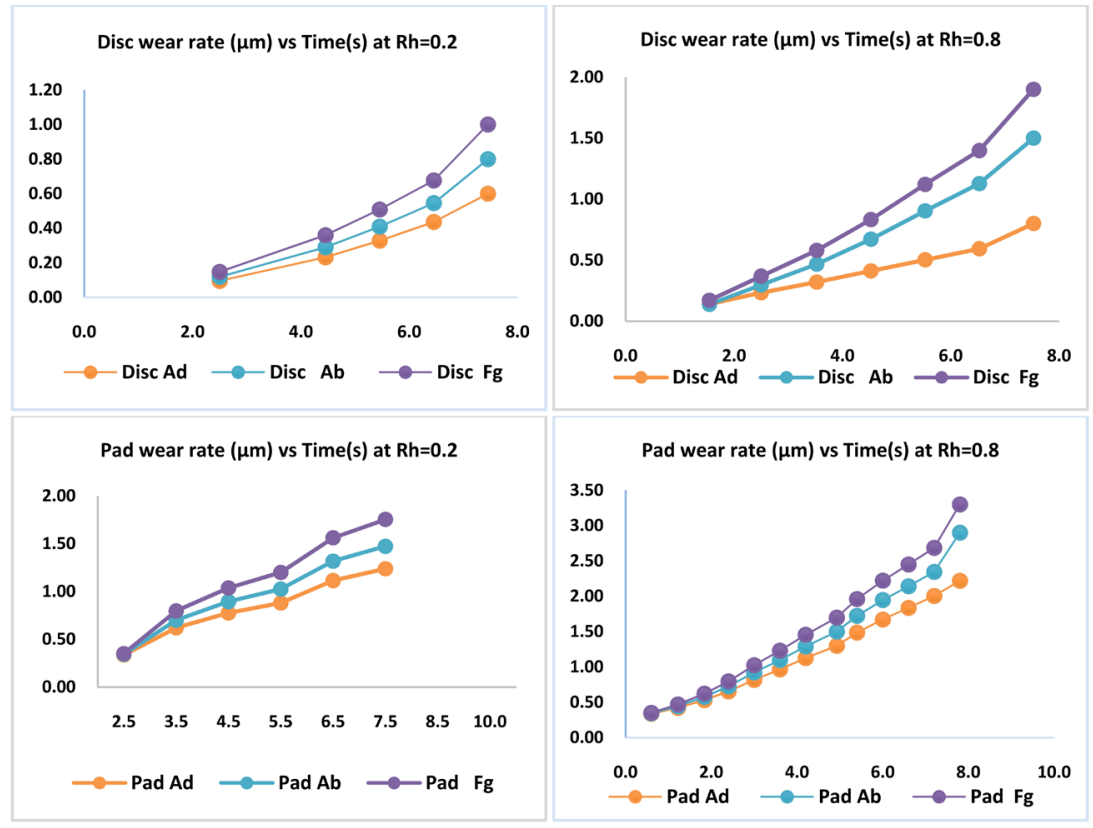

Figure 10. Lining and disc rate of material removal during dry and wet braking scenarios.

The rate of material removal gradually reduces as the lining/disc contact pressure increases during dry braking scenarios and escalades in wet scenarios as contact pressure increases with velocity and over all brake time. The increment in train speed and contact pressure under wet braking scenarios accelerates the rate of wear between the lining/disc, due to heat increment within the process. The above issue makes the braking action delicate and difficult to manage in many field operation using human intelligences. To endure safe operating mode, a reliable tool that has the ability to discover, segregate and track the RUL of the lining/disc phase change using material removal as the damage entity is very important because the material used in constructing brake lining is designed to wear faster than that of the brake disc.

\subsubsection{Feature Development Module}

Feature is a familiar term used in the context of condition-based maintenance of industrial systems as it concerns failure discovery and extraction in one sector and failure speculation through the establishment of remaining useful life in the other sector. As diagnostic is a static segregation of fair from unfair scenarios on industrial system, the term prognostics is centered on an ongoing segregated change of attitude as time evolves in the same system. Setting apart as excerpted and first rated features, they watch, appraise and speculate the robustness of the system. It contributes in excerpting hidden information found in natural signals using significant techniques such as prognosability, monotonibility and trendability [19]. The choice of technique for feature first rating relies on the deteriorating attitude of the given component or system. Some practical applications may call for the blinding of more than one technique (hybrid) depending on the decrement or increment of the natural signal. Concerning our axis of orientation, 
the monotonibility technique will be valorized as the attitude of deteriorating signal in Figure 9, Figure 10 is presented.

The practical evaluation of degradation for most engineering components in contact relies on time interval of material removal and tiredness. Secondly each component of a system presents its own damage signature. These paradoxes make life difficult to manage fault segregation and the attitude of failure evolution. The quality of any deteriorating signature must center on high esteem features obtained either from sensors or physics laws through residual information about the component or full system. As a dynamic and stochastic nature, the contact attitude of the lining/disc during field operation relies on high esteem signals to speculate their health conditions. The quality of the excerpt and first rated features is the aspiration of attentiveness. The drifting information gained through residual exploitation must partially be segregated from excerpted and first rated features in learning and testing categories.

Features are excerpted in stages, residual signals are gained based on variation within threshold signatures and the signatures obtained as the system deteriorates over time. Reliable, operating, surrounding and motion data sets are the aspiration of concern in time domain. They include characteristics of the lining/disc braking; rate of material removal, train speed, contact pressure, braking time; relative humidity, dust, lining factor; loads evolution, stiffness, damping and distance travel. These data's are merged in Equation (1) and redefined in Equations $(8 \& 9)$ to creates excerpted features based on the below remainder $\left(\% \tau_{Z W}\right.$ and $\left.\% \tau_{z U}\right)$ Equations (10 \& 11). Where, the suffix $z=1,2,3$ demonstrate the method of interest (model or data oriented) $t_{n}$ being the time increment for every $n$ braking action of the rail vehicle.

$$
\begin{gathered}
\% \tau_{Z W}(t)=\left(\left(\frac{\Delta\left(t_{n}\right)-\Delta\left(t_{n+1}\right)}{\Delta\left(t_{n}\right)}\right) * 100\right)_{z} \\
\% \tau_{Z U}(t)=\left(\left(\frac{t_{n}-t_{n+1}}{t_{n}}\right) * 100\right)_{z}
\end{gathered}
$$

First rating feature is the next vibrant issue to be addressed, as not all available features created at the excerpted stage is favorable to speculate system safe mode through deterioration signatures. To speculate the rate of material removal and balance safe mode of the lining/disc mounted on a CC2600 locomotive, Equation (12 \& 13) grant the permission. They expresses an absolute reduction/increment on the first rated feature patterns as time escalates, with $\aleph_{s}$ the number of trips per time interval that the lining/disc under full usage is obtained. After esteem extraction and first rating, the features are later segregated from learning and testing sets of information in the ratio (70:30). Learning features are used to develop the speculating signatures, meanwhile the testing set of information are used to check the goodness of the learning signatures before practical approval. 


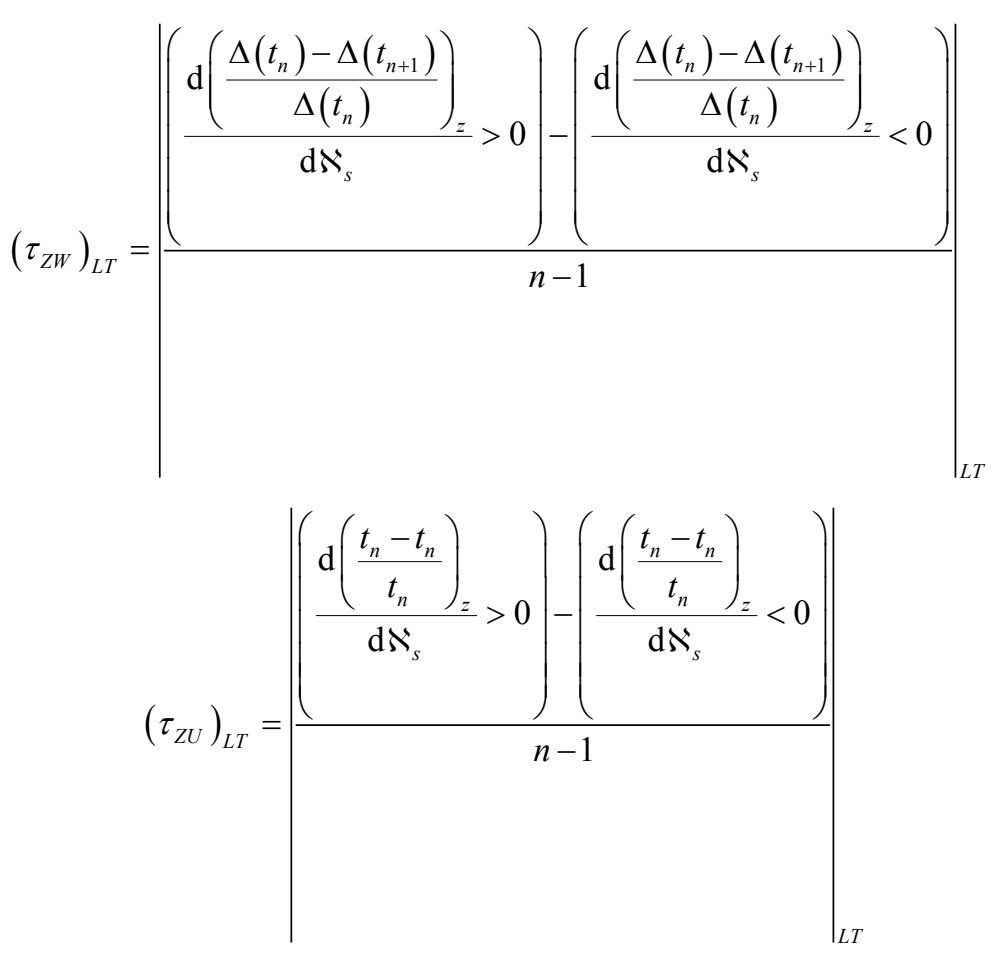

\subsubsection{ANFIS Modeling Module}

Based on the features gained after the excerpted and first rating stages, this module is segregated as ANFIS $_{Z w^{*}}$ for the rate of material removal speculation and ANFIS $_{Z U}$ : for remaining safe life speculation of the lining and disc respectively. For the case of ANFIS $_{Z W}$, the speculated signature for the rate of material removal for the lining/disc is examined with Equations (14-15). These equations favor the calculation of the average rate of material removal and the corresponding remainder gained from speculation using digital signatures. Equations (16-17) are used to investigate the remainder of the calculated signatures from the speculated ones in order to distinguish the safe mode of operating the lin-

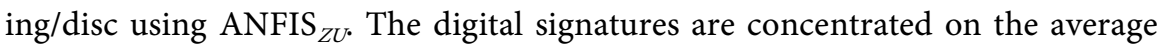
calculated time consumed or still to be consumed by individual components with the inconsistency rate of material removal (degradation signature). Figure 11(a), Figure 11(b) is the lining/disc personal calculated damage signatures investigated from equation (17), with the safe operating time of individual component calculated in time (seconds) domain and the rate of material removal (millimeters) respectively. From these signatures one can identify that the brake lining is designed to wear faster than the disc as their calculated safe modes differ from $67.4 \times 10^{4} \mathrm{~s}$ (8 years) for the lining, regarding $24.2 \times 10^{8} \mathrm{~s}$ (29 years) for the disc. Even though the degradation value for the lining $(20 \mathrm{~mm})$ is three times greater contrast to that of the disc $(7 \mathrm{~mm})$ on a single side of contact (Figure 4) while the safe life parameter drops continuously as the rate of damage improve. Where: $T_{m}$ lining/disc maximum millage to cover when the train is under operations; $T_{u}$ maximum millage already covered; $B_{s}$ maximum braking distance per trip and $T_{s}$ total braking point per trip. Table 2 below is the adopted parameters 
used in our current situation as not all the railway networks and trips to be performed by individual trains are the same. Let CC2600 locomotive rail vehicle on Douala-Yaoundé railroad network is valorised in these current exercises (TRANSCAM 1).

$$
\begin{gathered}
\Delta \varphi_{l / d}=\sum\left(\frac{\varphi_{a b}+\varphi_{a d}+\varphi_{f}}{m}\right)_{l / d} \\
\operatorname{Reminder}\left(\tau_{Z W}\right)=\left(\frac{\operatorname{Calculated}\left(\Delta \varphi_{l / d}\right)-\operatorname{speculated}\left(\Delta \varphi_{l / d}\right)}{\operatorname{Calculated}\left(\Delta \varphi_{l / d}\right)}\right) * 100 \% \\
\operatorname{Reminder}\left(\tau_{Z U}\right)=\left(\frac{\operatorname{Calculated}\left(R U L_{l / d}\right)-\operatorname{speculated}\left(R U L_{l / d}\right)}{\operatorname{Calculated}\left(R U L_{l / d}\right)}\right) * 100 \% \\
R U L_{l / d}=\left(\frac{T_{m}-T_{u}}{B_{s} * T_{s} * \aleph_{s}} * 24 x * 3600\right)_{l / d} \operatorname{seconds}(\mathrm{s})
\end{gathered}
$$

Table 2. Adopted operating framework for the railroad.

\begin{tabular}{rccccc}
\hline Ref. & $T_{m}(\mathrm{~km})$ & $T_{u}(\mathrm{~km})$ & $B_{s}(\mathrm{~km})$ & $T_{s}$ & $\aleph_{s}$ \\
\hline Disc & 1.1 million & 0 & 0.84 & 17 & 2 \\
Lining & 80,000 & & & \\
\hline
\end{tabular}

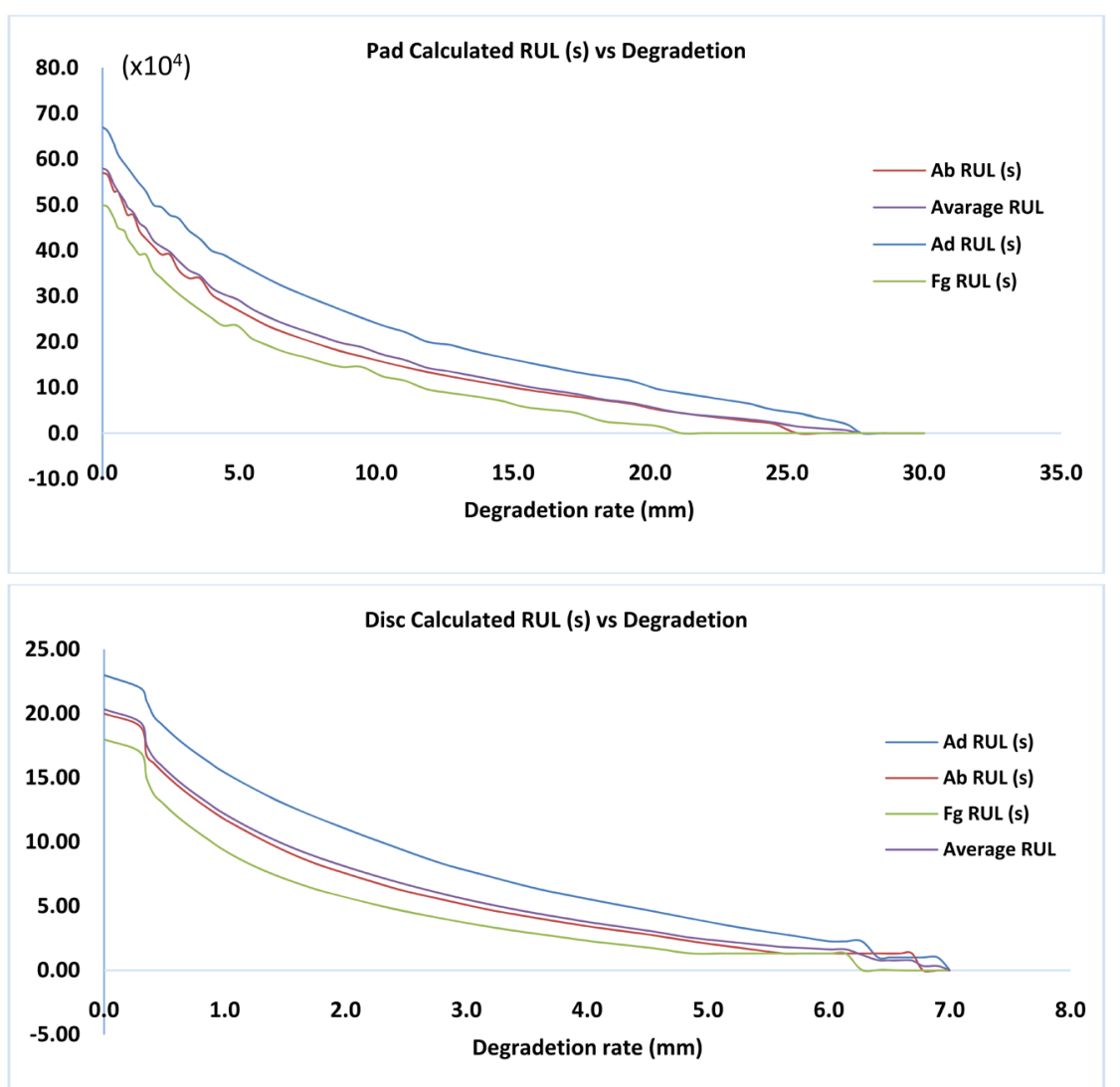

Figure 11. Calculated RUL for the CC2600 brake lining/disc. 
Regarding the speculating rate of material removal (wear) for the lining/disc under manipulation, digital signals such as train speed, contact pressure and train braking duration were the aspiration of attentiveness in learning and authenticating the accepted $\mathrm{ANFIS}_{Z W}$. Designed as a single input layer with three aspiring digital inputs, the model valorises bell-shaped membership functions as output linguistic information of the first layer. These types of membership functions accept the nonlinearity attitude showcased during train braking (when the lining/disc are in contact). In practice, the contact pressure varies during braking and thus leads to inconstant rate of material removal for the lining/disc. During learning of the model, the attitude of these membership functions are inconstant as the main input values of the digital signals show cases a vagrant attitude in real time. The rules are linked and adjusted to their individual node through the addition of their firing strength in the second and third layers using the gradient decent edge near in a reverse manner. The aggregated output signal which is centered on the addition and multiplication of the first and second rules demonstrate the forth layer, while the duty of the last layer is to aggregate a final output of the model using add-up features.

The learning in these layers is updated using the least square edge near in a forward manner. The accepted ANFIS $_{Z W}$ structure used for speculating the rate of material removal for lining/disc at this stage was chosen by keeping down the remainder between calculated and speculated outputs. Made of three input signals and a single output, the structure showcases 78 hidden nodes after being leant. A total of 108 linear and 27 nonlinear criteria was gained using a series of 5 learning pairs and 27 fuzzy precepts. Figures 12(a)-(d) are the dissimilar significant behavior of the input signals in relative to the output, with velocity showcasing a very high influence on the rate of material removal under dry and wet conditions. The increment in velocity thus increases the braking time which is a key factor in managing railroad traffic as it thus increases the braking distances which is an object of minimization in railway traffic management in Cameroon and other parts of the world. The vagrant braking distance in most cases leads to derailment and damage of railway infrastructures which affect the cost of production in this sector (the 2016 Eseka derailment in Cameroon). The learnt model was later authenticated using the percentage ratio of 70:30 for learning and authenticating signatures as mentioned above. Figure 13 is an extract of the model calculated output and speculated out with respect to its input signatures (Figures 12(a)-(d)). The rate of material removal under wet scenario showcases a very high significant digital signatures of (33.86 \& 32.80; $13.92 \&$ 12.63) $\mathrm{mm}$ contrast to $(17.74 \& 16.27 ; 6.80 \& 6.26) \mathrm{mm}$ for dry and average scenarios. This significance is clear to understand that wet braking circumstances have a high impact in the design and maintenance phase of the lining and disc. The perfection of the speculated ANFIS $_{Z W}$ model for material removal was gained using Equations $(17 \& 20)$ as portrayed in Table 3, where $\tau_{Z W A}$, is the model perfection and $S$ the number of digital signatures authenticated $(S=10)$. 
The maximum remainders gained after ANFIS $_{Z W}$ speculation for the lining/disc are (7.33 \& 9.99)\% respectively, and thus its perfections are (95.59 \& 94.11)\% respectively.

These shallow remainders are a clue that guarantees the goodness of the model between calculated and speculated output signatures in digital form. The quality of perfection for the initiated ANFIS $_{Z W}$ showcases the ability of speculating lining/disc material removal in real time.

$$
\tau_{Z W A}=S^{-1} \sum_{j-1}^{S}\left(1-\operatorname{Reminder}\left(\tau_{Z W}\right)\right) * 100 \%
$$
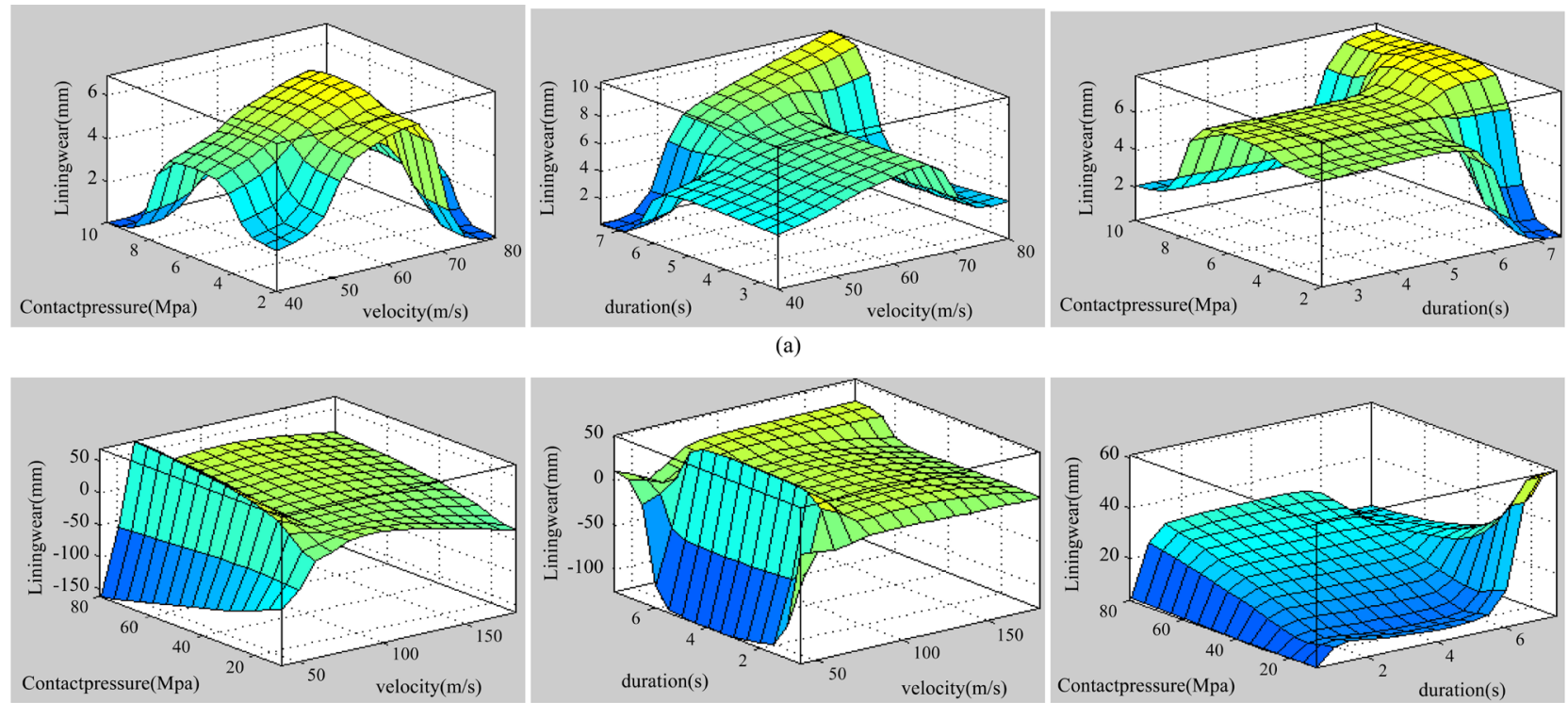

(b)
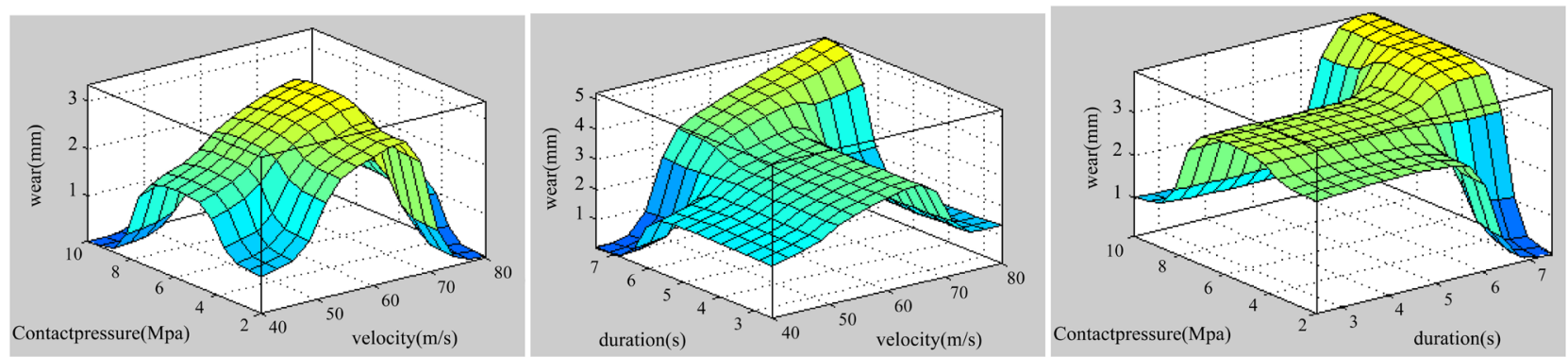

(c)
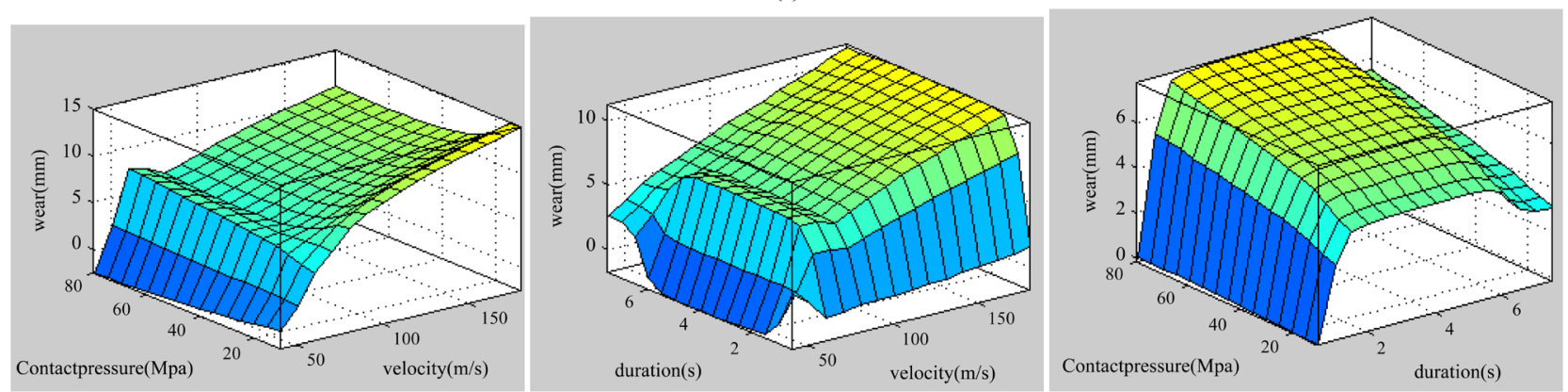

(d)

Figure 12. Attitude of ANFIS $_{Z W}$ inputs variables: $(\mathrm{a}-\mathrm{b})$ for the lining dry and wet condition, (c - d) for the disc dry and wet condition. 


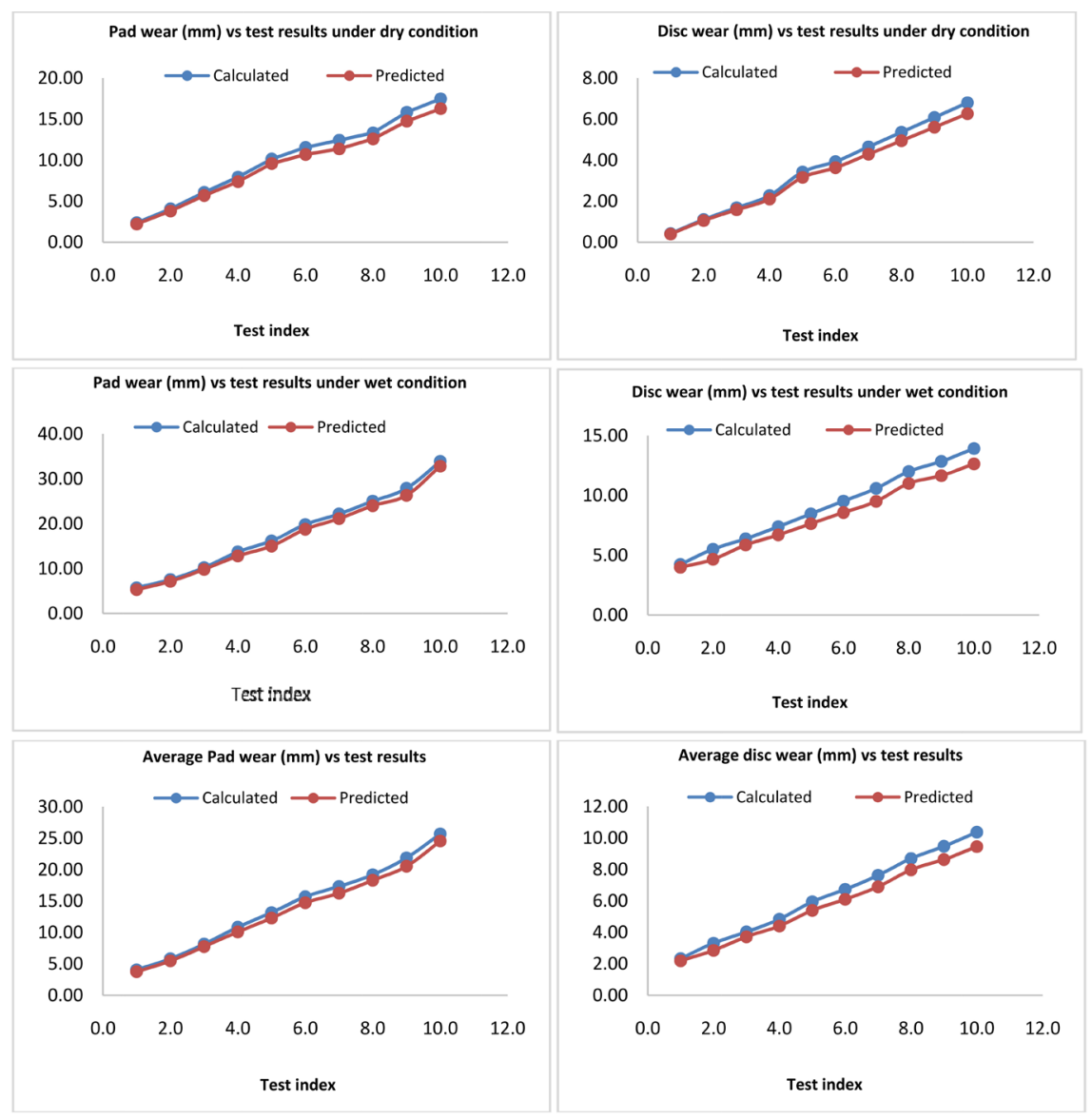

Figure 13. Authenticating calculated from speculated (ANFIS $Z_{W W}$ ) rate of material removal for the lining/disc.

Table 3. Characteristics of the suggested ANFIS $_{Z W}$ model perfection and error.

\begin{tabular}{ccccccccc}
\hline \multirow{2}{*}{$\begin{array}{c}\text { Test } \\
\text { index }\end{array}$} & \multicolumn{5}{c}{ Pad } & & \multicolumn{5}{c}{ Disc } \\
\cline { 2 - 8 } & Calculated & Predicted & Error & Accuracy & Calculated & Predicted & Error Accuracy \\
\hline 1.0 & 4.05 & 3.76 & 7.33 & $\mathbf{9 2 . 6 7}$ & 2.33 & 2.19 & 5.89 & $\mathbf{9 4 . 1 1}$ \\
2.0 & 5.82 & 5.47 & 5.96 & $\mathbf{9 4 . 0 4}$ & 3.30 & 2.85 & 9.99 & $\mathbf{9 0 . 1}$ \\
3.0 & 8.15 & 7.74 & 4.97 & $\mathbf{9 5 . 0 3}$ & 4.02 & 3.72 & 7.64 & $\mathbf{9 2 . 3 6}$ \\
4.0 & 10.82 & 10.08 & 6.82 & $\mathbf{9 3 . 1 8}$ & 4.83 & 4.40 & 8.91 & $\mathbf{9 1 . 0 9}$ \\
5.0 & 13.16 & 12.29 & 6.62 & $\mathbf{9 3 . 3 8}$ & 5.94 & 5.39 & 9.17 & $\mathbf{9 0 . 8 3}$ \\
6.0 & 15.68 & 14.72 & 6.16 & $\mathbf{9 3 . 8 4}$ & 6.73 & 6.10 & 9.35 & $\mathbf{9 0 . 6 5}$ \\
7.0 & 17.31 & 16.24 & 6.16 & $\mathbf{9 3 . 8 4}$ & 7.62 & 6.89 & 9.52 & $\mathbf{9 0 . 4 8}$ \\
8.0 & 19.17 & 18.29 & 4.61 & $\mathbf{9 5 . 3 9}$ & 8.68 & 7.97 & 8.16 & $\mathbf{9 1 . 8 4}$ \\
9.0 & 21.85 & 20.52 & 6.09 & $\mathbf{9 3 . 9 1}$ & 9.46 & 8.63 & 8.85 & $\mathbf{9 1 . 1 5}$ \\
10.0 & 25.66 & 24.53 & 4.41 & $\mathbf{9 5 . 5 9}$ & 10.36 & 9.45 & 8.82 & $\mathbf{9 1 . 1 8}$ \\
\hline
\end{tabular}

The speculation of the remaining useful life $\left(\mathrm{ANFIS}_{Z U}\right)$ for the lining/disc is the next episode of attentiveness as it favors the smooth monitoring of the sys- 
tem by maintenance agents and rail vehicle operators. In line with the aspirations of ANFIS $_{Z W}$ for material removal, ANFIS $_{Z U}$ integrates digital signals such as train speed, contact pressure and rate of material removal as input signatures for learning and authenticating of the model. The model is designed as a single input layer with three aspiring digital inputs signatures and valorised with bell-shaped membership functions. The duty of the last layer is to aggregate a final output of the model using add-up features. The learning in these layers is updated using the least square edge near in a forward manner.

The accepted ANFIS $_{Z U}$ structure used for speculating the remaining useful life (RUL) for lining/disc at this stage was chosen by keeping down the remainder between calculated and speculated output signatures. Made of three input signals and a single output, the structure showcases 78 hidden nodes after being learnt. A total of 108 linear and 27 nonlinear criteria were gained using a series of 52 learning pairs and 27 fuzzy precepts. Figure 14(a), Figure 14(b) are the dissimilar significant behavior of the input signatures in relative to the output, with the rate of material removal showcasing a very high influence on the safe mode for both lining and the disc. The braking velocity and contact pressure has a huge significance on the rate of material removal as mentioned above. For attentiveness, the damage signature shall be centered on the rate of material removal and the duration of complete usage of the component in real time. This phenomena is very important to freight trains and passenger coaches because they don't have to holdout gravely in acute usage, as the braking system in general holds an important safety factor during train operation. The regular control of the lining/disc is undoubtedly critical every moment the operator or maintenance agent are engaged in usage of the train. But unfortunately, it is not possible

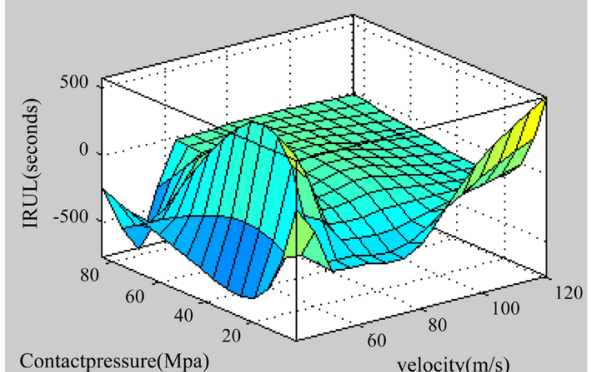

velocity $(\mathrm{m} / \mathrm{s})$
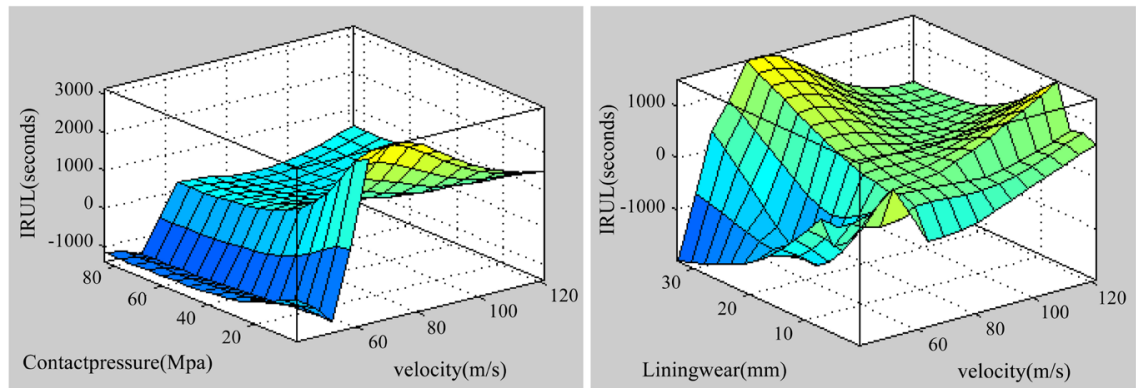

(b)
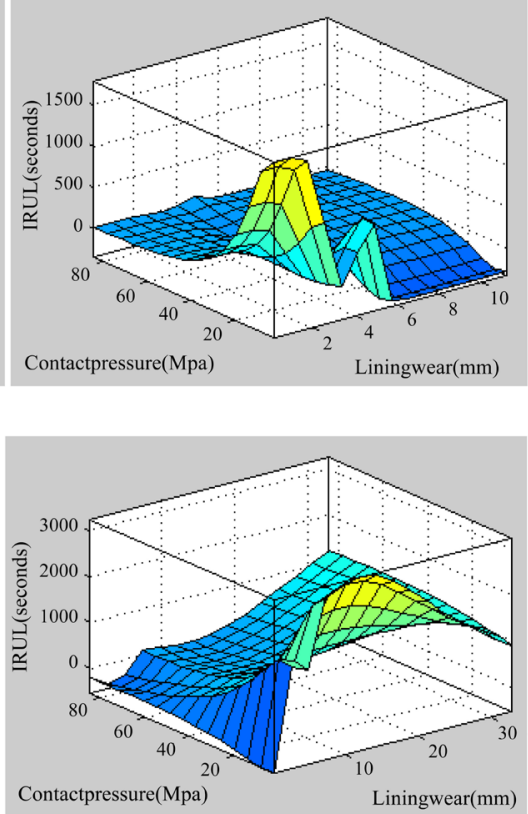

Liningwear(mm)

Figure 14. Attitude of ANFIS $_{Z U}$ inputs variables: (a) for the lining, (b) for the disc. 
to make any customary declaration on when the required adjustment or full substitution, when they are fully worn out, and thus their service life respectively. These issues can be remedied through speculating their remaining safe life, which is the object of attentiveness in this episode.

To speculate the RUL of the system, authenticating the learnt model ( $\mathrm{ANFIS}_{Z U}$ ) is the object of attentiveness. The ability to make-out a forethought damaged threshold signature for speculating the ANFIS $_{Z U}$ model output is relevant using the percentage ratio of 70:30 for both scenarios one at a time. Figure 15 is an extract that showcases the model calculated output signatures from the speculated output signatures in alignment with their input signatures (Figure 14(a), Figure 14(b)).

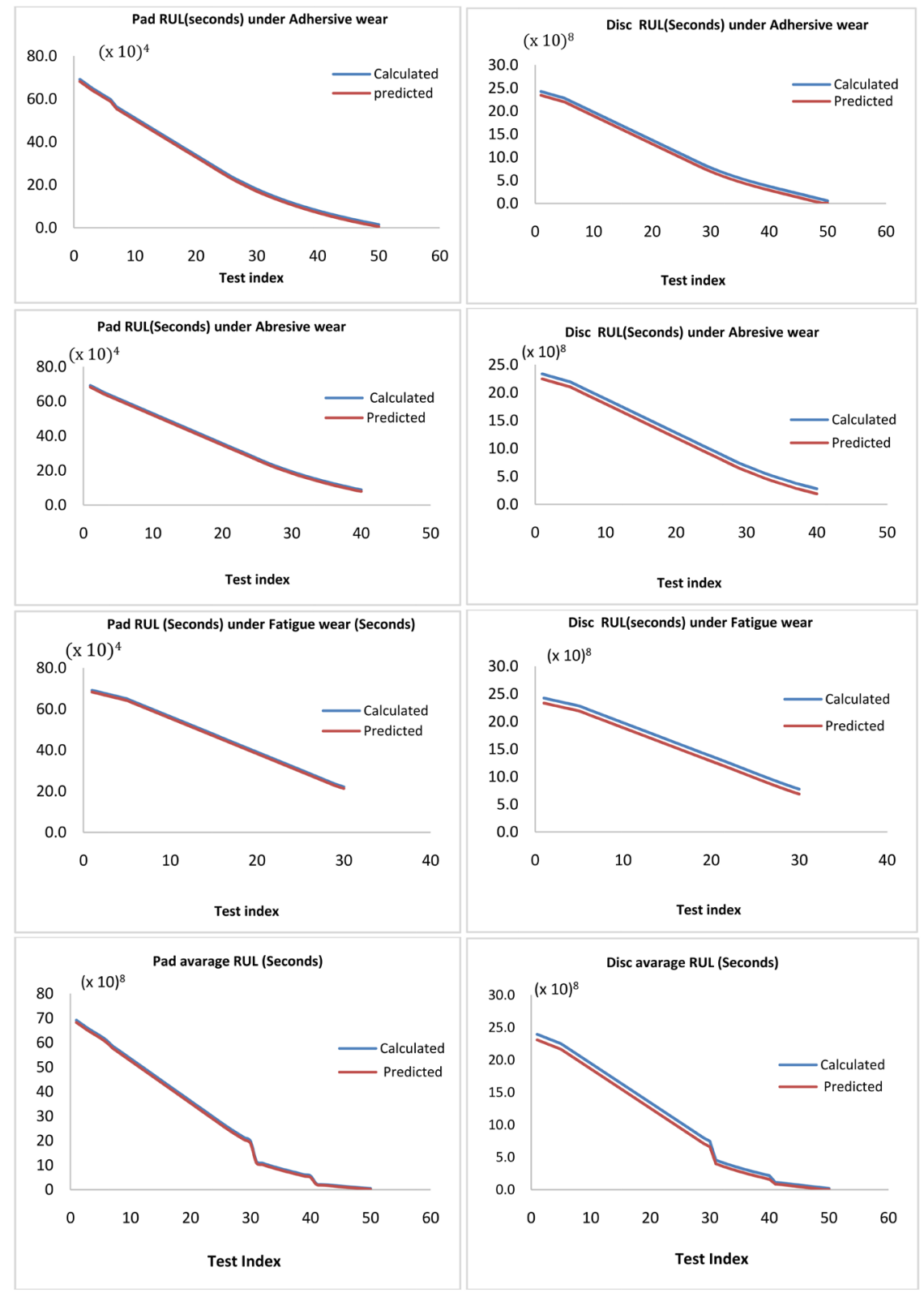

Figure 15. Authenticating calculated from speculated $\left(\mathrm{ANFIS}_{Z U}\right)$ RUL for lining/disc. 
In general, awareness shows that the average calculated RUL and speculated RUL curve showcase a significant damage signature of the lining/disc under investigation as in real time exploitation with little remainder (Equation (21)). But specifically, the lining is designed to be replaced often as contrasted to the disc during usage; they are engaged under one or a combination of two wear scenarios with unique damage signature. Their RUL output calculated and speculated digital signatures differ (Table 4) with fatigue scenarios leading the trend. These digital signatures demonstrate how server continues follow up of the braking operation is necessary to the rolling stock industry as mechanical failures are concerned. The failure of the braking operations indirectly account to about 75\% failures of the wheelset and chassis as under braking or over braking thus contribute to surface initiative defects and fragments on the brake disc, pads and on wheels' rail contacts

To perfect the above speculated model for remaining useful life $\left(\mathrm{ANFIS}_{Z U}\right)$, Equations $(17 \& 21)$ are considered with their results portrayed and interpreted in Table 5, where $\tau_{Z U A}$, is the model perfection and $S$ the number of authenticated digital signatures $(S=10)$. The maximum remainders gained after ANFIS $_{Z U}$ speculation for the lining/disc are $(9.98 \& 6.37) \%$, and its perfections are $(96.82 \&$ $96.37) \%$ one at a time. These shallow remainders are a clue that guarantee the

Table 4. Summary of lining/disc RUL under different type of material removal.

\begin{tabular}{|c|c|c|c|c|c|c|c|c|}
\hline \multirow{3}{*}{ Wear Type } & \multicolumn{4}{|c|}{ Pad } & \multicolumn{4}{|c|}{ Disc } \\
\hline & \multicolumn{2}{|c|}{ RUL $\times 10^{4}$ (seconds) } & \multicolumn{2}{|c|}{ Maximum $\varphi_{I}(\mathrm{~mm})$} & \multicolumn{2}{|c|}{ RUL $\times 10^{8}$ (seconds) } & \multicolumn{2}{|c|}{ Maximum $\varphi_{\mathrm{d}}(\mathrm{mm})$} \\
\hline & Calculated & speculated & Calculated & speculated & Calculated & speculated & Calculated & speculated \\
\hline Abrasive & 66.60 & 65.64 & 19.80 & 19.68 & 21.00 & 20.20 & 7.0 & 6.97 \\
\hline Adhesive & 56.40 & 53.70 & 19.50 & 19.46 & 17.40 & 16.20 & 6.9 & 6.85 \\
\hline Fatigue & 47.70 & 46.90 & 19.30 & 19.28 & 14.90 & 14.00 & 6.8 & 6.76 \\
\hline
\end{tabular}

Table 5. Characteristics of the suggested ANFIS $_{Z U}$ model perfection and error.

\begin{tabular}{|c|c|c|c|c|c|c|c|c|}
\hline \multirow{2}{*}{ Test index } & \multicolumn{4}{|c|}{ PAD } & \multicolumn{4}{|c|}{ DISC } \\
\hline & Calculated & Predicted & Error & Accuracy & Calculated & Predicted & Error & Accuracy \\
\hline 5.0 & 62.72 & 60.73 & 3.18 & 96.82 & 23.94 & 23.07 & 3.63 & 96.37 \\
\hline 10.0 & 53.51 & 51.20 & 4.31 & 95.69 & 21.90 & 21.03 & 3.97 & 96.03 \\
\hline 15.0 & 44.87 & 42.87 & 4.45 & 95.55 & 18.87 & 18.00 & 4.30 & 95.70 \\
\hline 20.0 & 36.23 & 34.23 & 6.15 & 93.85 & 15.84 & 14.97 & 4.64 & 95.36 \\
\hline 25.0 & 27.59 & 25.59 & 7.61 & 92.39 & 12.81 & 11.94 & 4.98 & 95.02 \\
\hline 30.0 & 19.79 & 18.49 & 6.55 & 93.45 & 9.78 & 8.91 & 5.32 & 94.68 \\
\hline 35.0 & 8.60 & 7.57 & 8.14 & 91.86 & 4.55 & 3.98 & 5.66 & 94.34 \\
\hline 40.0 & 5.61 & 5.10 & 9.08 & 90.92 & 3.09 & 2.52 & 5.99 & 94.01 \\
\hline 45.0 & 1.48 & 1.33 & 9.98 & 90.02 & 1.12 & 0.86 & 6.33 & 93.67 \\
\hline 50.0 & 0.50 & 0.45 & 9.35 & 90.65 & 0.61 & 0.34 & 6.67 & 93.33 \\
\hline
\end{tabular}


goodness of the model between calculated and speculated output signatures in digital form. Even though the quality of perfection showcases a decrement attitude (Table 5) due to the stochastic nature of the lining/disc contact scenario when the brake is energised, the initiated ANFIS $_{Z U}$ showcases the goodness of speculating lining/disc remaining safe operating mode in real time interval after 50 mode authentications as the safe life parameter drops continuously as the rate of damage improves.

$$
\tau_{Z U A}=S^{-1} \sum_{j-1}^{S}\left(1-\operatorname{Reminder}\left(\tau_{Z U}\right)\right) * 100 \%
$$

\subsubsection{Output Display and Online Storage Modules}

In practice, the storage and display modules are interconnected in a single electronic device. The main ideology of these modules is to segregate and safeguard the relevant operational input-output signatures required by the rail vehicle operator and the maintenance agent for smooth observation of the lining/disc in digital and linguistic shapes. It shall constitute a display screen mounted on the dashboard of the rail vehicle and the control room of individual train stations for distance observations. Lastly, an online display features designed to track and observe the same attitude using hardware, software and wireless technologies. The type of output signatories shall include; 0 or 1 for possible degradation localization, $\lambda_{l / d} \geq 1 \mathrm{~mm}$ for lining/disc penetrating distance; $\varphi_{l / d} \geq 0 \mathrm{~mm}$ for calculated, predicted and field recording rate of material removal digital signatures (example in Table 2 \& Table 3); $L_{R U L c}=L_{S p}, L_{R U L p} \leq L_{S p}$ and $d_{\text {RULc }}=L_{S d}, d_{R U L p} \leq L_{S d}$ for calculated, predicted and field recording rate of safe operating mode of the lining/disc digital signatures (example in Table 3 \& Table 4) with $L_{S p} \& L_{S p}$ the life span of the lining/disc one at a time; $\left[\varphi_{l / d}\right] \geq\left[\varphi_{l / d}\right]_{D D}$ for the contrast between calculated and field recorded rate of material removals and $\left[L_{R U L C}\right]_{M B} \geq\left[L_{R U L}\right]_{D D}$ for the lining/disc remaining safe operating modes. The field recording output signatures are not presented here as the next episode of this research shall capitalize on the design and development of a failure discovery, extraction and degradation field recorder for real time alert and observation of the braking units as the rate of material removals and operational safe modes are concerned. With such effect, the remainder between the model oriented and data oriented $(M B \neq D D)$ is not showcased in this section and thus the accuracy of suggested methodology to failure diagnostic and forecasting is still to be fully guaranteed. Regarding the online storage, the acquired analogues field recording signatures gained from interconnected sensors are transformed to digital signatures and stored online using iCloud technology. This shall be considered with reason being that the operating state of the lining/disc can be accessed by the operator, maintenance agent, company owners, researchers and academicians for further follow up and development of new theories in managing failures and repairs. The auto verification of the sensors and the monitoring device is required on the other hand, as their malfunctioning can also affect the attitude with which the system can be interpreted by the rail 
vehicle activists.

\section{Conclusion}

This research episode has focused on the development of a suggested model for failure forecasting using artificial intelligence technology. The model has been segregated as failure forecaster for the rate of material removal (ANFIS ${ }_{Z W}$ ) and an estimator for the remanant useful $\left(\mathrm{ANFIS}_{Z U}\right)$ working mode of the lining/disc. The rate of material removal and RUL under fatigue, adhesive and abrasive scenarios has been calculated during dry and wet braking conditions. The rate of material removal due to fatigue presents a very high trend contrast to adhesive or abrasive conditions. To ensure effective condition based maintenance, the speculated models were learnt and authenticate using natural threshold signatures and unnatural attitudes of degradation signatures initiated during simulation study. The quality and perfection of the suggested methodology has been achieved in accordance with the shallow remainder gained and the output after authentication. Regarding future authentication of this research, these results shall be used to develop an intelligent maintenance tool for active and proactive health management through data recording in the rolling stock industry.

\section{Conflicts of Interest}

The authors declare no conflicts of interest regarding the publication of this paper.

\section{References}

[1] ISO 13381-1 (2015) Condition Monitoring and Diagnostics of Machines-Prognostics-Part 1: General Guidelines.

[2] Luo, J., Namburu, M., Pattipati, K. and Qiauio, L. (2003) Model-Based Prognostic Techniques. IEEE Systems Readiness Technology Conference, Anaheim, 22-25 September 2003. https://www.teamqsi.com/wp-content/uploads/2012/10/063Luo.pdf

[3] Zhang, X.H., Xiao, L. and Kang, J.S. (2015) Degradation Prediction Model Based on a Neural Network with Dynamic Windows. Sensors, 15, 6996-7015. https://doi.org/10.3390/s150306996

[4] Wu, Q.H., Ding, K.Q. and Huang, B.Q. (2018) Approach for Fault Prognosis Using Recurrent Neural Network. Journal of Intelligent Manufacturing, 31, 1621-1633.

[5] Zid, K., Ahmed, M.B. and Turki, M. (2018) Modeling of Flank Wear Using ANFIS. Proceedings of the 4th International Conference on Engineering \& MIS, Istanbul, June 2018, Article No. 47.

[6] Sparthan, T., Nzie, W., Sohfotsing, B., Garro, O. and Beda, T. (2020) A Proposed Scheme for Fault Discovery and Extraction Using ANFIS: Application to Train Braking System. Studies in Engineering and Technology, 7, 48. https://doi.org/10.11114/set.v7i1.4822

[7] Jaoude, A.A. (2012) Advanced Analytic Model for the Prognostic of Industrial Systems Subject to Fatigue. PhD Thesis.

[8] Chong, S.Y., Lee, J.-R. and Shin, H.-J. (2010) A Review of Health and Operation Monitoring Technologies for Trains. Smart Structures and Systems, 6, 1079-1105. 
https://doi.org/10.12989/sss.2010.6.9.1079

[9] Sawczuk, W. and Szymanski, G.M. (2017) Diagnostics of the Railway Friction Disc Brake Based on the Analysis of the Vibration Signals in Terms of Resonant Frequency. Archive of Applied Mechanics, 87, 801-815.

https://doi.org/10.1007/s00419-016-1202-0

[10] Feildmanis, J. (2008) Mathematical Modeling of Disc Brake Frictional Lining Heat and Wear. Engineering for Rural Development, Jelgava, 239-241.

http://www.tf.llu.lv/conference/proceedings2008/Papers/45_Feldmanis.pdf

[11] Kakad, S.R., More, R.M. and Kamble, D.N. (2017) Mathematical Modeling \& Analysis of Brake Pad for Wear Characteristics. International Conference on Ideas, Impact and Innovation in Mechanical Engineering, 5, 1048-1056.

[12] Kalhapure, V.A. and Khairnar, H.P. (2018) Wear Mechanism and Modelling for Automotive Brakes with Influence of Pressure, Temperature and Sliding Velocity: A Review Article. European Journal of Advances in Engineering and Technology, 5, 333-343.

[13] Stoica, N.A., Petrescu, A.-M. and Tudor, A. (2018) Modelling the Wear Processes of the Automotive Brake Pad and Disc. INCAS Bulletin, 10, 169-179.

https://doi.org/10.13111/2066-8201.2018.10.4.15

[14] Sawczuk, W. and Tomaszewski, F. (2013) Evaluation of the Wear of Friction Pads Railway Disc Brake Using Selected Point Parameters of Vibrations Signal Generated by the Disc Brake. Diagnostyka, 14, 69.

[15] Sawczuk, W. (2017) The Application of Vibration Accelerations in the Assessment of Average Friction Coefficient of a Railway Brake Disc. Measurement Science Review, 17, 125-134. https://doi.org/10.1515/msr-2017-0016

[16] Dineshkumar, S. and Jayakanth, J.J. (2016) Modelling and Analysis of Disc Brake. International Journal of Innovative Research in Science, Engineering and Technology, 5, 5403.

[17] Stoica, N.A. (2018) Contributions to the Study of the Tribological Processes in the Disc Braking System, Applied to Automobiles. Thesis, University Politehnica of Bucharest, Bucharest.

[18] CAMTECH (2014) Pamphlet on Instruction for Axle Mounted Brake Disc in LHB Coach (FTIL Type). CAMTECH, Maharajpur.

[19] Azevedo, D., Cardoso, A. and Ribeiro, B. (2020) Estimation of Health Indicators Using Advanced Analytics for Prediction of Aircraft Systems Remaining Useful Lifetime. European Conference of the Prognostics and Health Management Society, Turin, 27-31 July 2020, 1-10. 